\title{
MEDIUM ENERGY \\ NUCLEAR PHYSICS RESEARCH
}

\author{
Progress Report \\ for the period July 1, 1987 - September 30, 1988 \\ DOE/ER/40515-7
}

\section{G.A. Peterson, J.F.Dubach, R.S. Hicks, and R.A. Miskimen University of Massachusetts Amherst, MA 01003}

September, 1988

Prepared for

The U.S. Department of Energy

Under Grant No. DE-FG02-88ER40415.A000

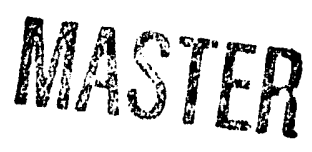


This report was prepared as an account of work sponsored by the United States Government. Neither the United States nor the United States Department of Energy, nor any of their employees, nor any of their contractors, subcontractors, or their employees, makes any warranty, expressed or immplied, or assumes any legal liability of responsibility for the accuracy, completeness, or usefulness of any information, apparatus, product, or process disclosed, or represents that its use would not infringe privately owned rights. 


\section{INTRODUCTION}

During the past 14 months since the last progress report, the University of Massachusetts Medium Energy Nuclear Physics Group has continued its work in nuclear structure physics, but it has also pursued new endeavors at higher momentum transfers, and has been involved in the planning of future facilities. The group is in the process of making a transition from nuclear structure physics to work that bears upon the quark substructure of nucleons. This new class of experimental and iheoretical work should dominate tnuclear physics in the 1990's. It will involve higher energy probes and probably deal with few-nucleon systems.

The UMass group has concentrated on using electromagnelic probes, particularly the electron in high-energy scattering experiments at the Stanford Linear Accelerator Center (SLAC). F'lans are also being made for high energy work at the Continuous Beam Accelerator Facility (CEBAF). The properties of this accelerator should permit a whole new class of coincidence experiments to be carried out.

At SLAC UMass has made major contributions toward the plans for a cluster-jet gas target and detector system at the $16 \mathrm{GeV}$ PEP storage ing (EEGASYS). Associate Professor Ross Hicks spent his sabbatice' year at SLAC working on the design of the "Big Acceptance Dipole" (BAD) magnet for PEGASYS and on many other features of this project, as well as helping to prepare for NPAS experiment NE-11 on neutron and proton form factors. He was greatly aided by postdoctoral research assoclate Sayed Rokni who is stationed at SLAC. Rokni has made Monte Carlo simulations of expected events for many projected experiments with PEGASYS.

For the future EEBAF accelerator, UMass actually participated in soine related experimentad work even though the CEBAF beam is not expected until 1994. Tests were made of the feasibility of operating wire drift chambers in the vicinity of a continuous electron beam at the University of Illinols microtron. Assistant Professor Rory Miskimen; formerly from Illinois, and postdoctoral research associate Hosain Baghael, made measurements, which were later compared to state of the art rate calculations in order to check on how background events would affect a projected luminosity for the Large Acceptance Detector (LAS) at CEBAF. The University of Massachusetts also has submitted letters of intent for future experiments at CEBAF. 
At the same time a program of studies of the nuclear structure of more complex nuclei has been continued at the MIT-Bates Linear Accelerater Center and in Amsterdam at the NIKHEF-K laboratory. This experimental work, including that completed and that in progress, has been in cencert with and has been strongly supported by the theeretical work of Associate Professor John Dubach, postdoctoral research associate Gregory Crawtord, and their students.

At the MIT-Bates Accelerater, because of an unforeseen change in beam scheduling as a result of problems with the $T_{20}$ experiment, the UMass group was able to complete data acquisition on experiments involving $180^{\circ}$ elastic magnetic scattering on ${ }^{117} \mathrm{Sn}$ and $41 \mathrm{Ca}$. The latter experiment involved a collaboration with experimenters from Saclay.

A considerable effort has been given to preparations for a future experiment at Bates involving the high-resolution threshold electrodisintegration of the deuteron. Prof. Miskimen has constructed special drift chambers to be used in the focal "plane" of the ELSSY spectrometer. The use of these chambers should permit a high degree of discrimination against background events in the measurement of the almost neutrino-like small cross sections that are expected.

If time permits, the UMass group may submit two proposals to Bates for future work: one on a low counting rate measurement pertaining to cluster substructures in nuclel, and a second on an out-of-plane colncidence measurement of deuteron threshold electrodisintegration. This latter measurement will be made in hopes of obtaining the transverse-longltudinal interference term and will be made in collaberatien with physicists frem Japan. This UMass-Japan collaboration was started this past summer with the approximate month-long visit of four Japanese physicists to the University. The Japanese government has provided fundstor travel, etc., in the amount of about $\$ 100,000$ to support this collaboration for a three year period.

In Amsterdam at the NIKHEF-K facility, single arm $\left(\theta, \theta^{\prime}\right)$ measurements were made in November of 1987 on ${ }^{10_{B}}$ in order to better determine the $\mathrm{P}_{3 / 2}$ wave function from the transition from the $\mathrm{J}^{\pi}=3^{+}$ground state to the $0^{+}$excited state at $1.74 \mathrm{MeV}$. In June and July of 1988, $\left(\theta, \theta^{\prime} p\right)$ coincidence measurements on ${ }^{10} \mathrm{~B}$ were completed. The objectlve was to obtain information on the $\mathrm{P}_{3} / 2$ wave function by another means. If there is a disagreement between the two experiments, then perhaps aspects of the $\left(\theta, \theta^{\prime} p\right)$ reaction, such as final state interactions, can be elucidated.

*Funds paid directly to Japanese Physicists. 
In summary, the UMass Medium Energy Nuclear Physics Group has been engaged in a broad range of electromagnetic interaction nuclear physics projects during this reporting period. Several experimental and theoretical projects have been completed and the results have been published, complete data sets have been acquired on three experiments, and important new particle detection equipment has been built. At the same time a substantial effort has been made in planning and proposing future experiments at Bates, SLAC, and CEBAF. Designs of detectors and magnets are underway, and new collaborations have been formed.

This report will be organized as follows. Part II will discuss experimental work at the five laboratories where UMass has been involved: (1) Bates, (2) SLAC, (3) NIKHEF-K, (4) CEBAF, and (5) Illinois. Discussion will be broken down into work completed, work in progress, and work planned. Part III of this report discusses the theoretical work. Publications are listed in part IV. The active seminar and colloqulum program is described in part $V$, the status report on the VAX computer is given in part VI, comments on personnel are given in part VII, the possible need for new facilities is described in part VIII, new equipment obtained primarlly from funds from UMass is described in part IX, financial assistance other than that provided by the grant is described in part $X$, and comments on the budget are given in part XI. 


\section{EXPERIMENTAL WORK}

\section{A. Experiments at the Bates Linear Accelerator Center}

Our nuclear structure work at Bates has generally concentrated on p-shell or light nuclei, and on measurements of transverse cross sections, especially by $180^{\circ}$ scattering as the UMass group built the $180^{\circ}$ scattering magnet system. The relevance of our work to other areas of nuclear physics is manifest: electron scattering provides the benchmark of wave function determinations. These in turn provide the necessary information for the interpretation of less well-understood reactions involving probes such as pions and protons. We have emphasized work on Ilght nuclei because they are especially suitable for detailed comparisons with microscopic theories. Our experimental work on the light nuclei meshes nicely with the interests of UMass theorist John Dubach.

1. Experiments in Progress: Data Acquisition Incomplete

\section{a. Experiment 87-02: Threshold Electroexcitation of the Deuteron at Hiah}

\section{Mementum Transfer}

The electrodisintegration of the deuteron near threshold by backward angle electron scattering involves the interaction of transverse virtual photons with the current and magnetization densities of the deuteron. Within a few MeV of threshold, a spin-flip magnetic dipole transition from the isospin singlet ${ }^{3} S_{1}+{ }^{3} D_{1}$ ground state to the quasibound isospin triplet ${ }^{1} S_{0}$ state dominates the cross section. This transition can be thought of as a resonance, and gives a sharp rise or cusp in electron scattering cross sections just at threshold.

The transverse thrishold electrodisintegration of the deuteron involves the use of the simplest and best-understood reaction with the simplest nuclear target. Higher order effects, such as various meson exchange currents, can be investlgated without the complications surrounding the use of more complicated reactions. There have been experimental measurements of threshold electrodisintegration up to squared momentum transfers as high as $\mathrm{Q}^{2}=27.8 \mathrm{fm}^{-2}$ by Auffret et al. (Phys. Rev. Lett. 55, $1362(1985)$. We propose to approximately double the $Q^{2}$ range of these measurements to $Q^{2}=48 \mathrm{~mm}^{-2}$. 
The electrodisintegration of the deuteron at threshold provides one of our most important tests for the validity of the meson exchange representation for the nucleonnucleon force. As shown in the following figure, a calculation in the impulse approximation (i.e., for nucleons only) cannot account for the differential cross sections measured above squared four-momentum transfers of $Q^{2}=5 \mathrm{fm}^{-2}$. In particular, the data of Auffret show no evidence whatsoever for the deep diffraction minimum predicted to exist near $Q^{2}=12 \mathrm{fm}^{-2}$. duig to destructive interference between ${ }^{3} S_{1} \rightarrow{ }^{1} S_{0}$ and ${ }^{3} D_{1} \rightarrow{ }^{1} S_{0}$ transitions. The consideration of one-plon exchange currents is crucial for understanding the lack of a diffractive minimum in this $Q^{2}$ region.

Above $\mathrm{Q}^{2}=20 \mathrm{fn}^{-2}$ the interpretation of the data becomes less clear. At this point the theoretical predictions of Mathiot (Nucl. Phys. A412, 210 (1984)) which include pion exchange currents, begin to drop sharply below the observed cross sections. These predictions are also shown in the figure. However, with the additional inclusion of $p$ meson exchange and $\Delta_{33}$ admixtures in the wave functions, Mathlot's calculations are brought back into agreement with the data, at least as far as $Q^{2}=24 \mathrm{~mm}^{-2}$. Nevertheless, all existing theoretical predictions for $Q^{2}>20 \mathrm{fm}^{-2}$ are dependent upon hypotheses which have yet to be proven. For example, different arguments have been given for the use of either the Sachs nucleon isovector form factor $G_{E}$ or the Dirac form factor $F_{1}$ in the calculations. The results obtained for these two choices display large differences in this high $Q^{2}$ region.

It is the objective of this proposal to extend the kinematic range of data on the threshold electrodisintegration of the deuteron to $Q^{2}=48 \mathrm{~mm}^{-2}$. The availability of these data will certainly aid in defining the appropriate dynamic model for deuteron electrodisintegration. At this time, as shown in the next figure, large divergencles arise among the various theoretical predictions for $Q^{2}>30 \mathrm{fm}^{-2}$. New experimental information, when combined with other constraints, such as the new measurements of the deuteron elastic form factors, will lead to considerable improvements in our understanding of this fundamental nucleus.

This major experiment was approved in March of 1987 for 350 hours of beam time. The cross sections are expected to be extremely small at high momentum transfers, of the order of $10^{-40} \mathrm{~cm}^{2} / \mathrm{sr}$, and hence long counting times with low-background conditions are 


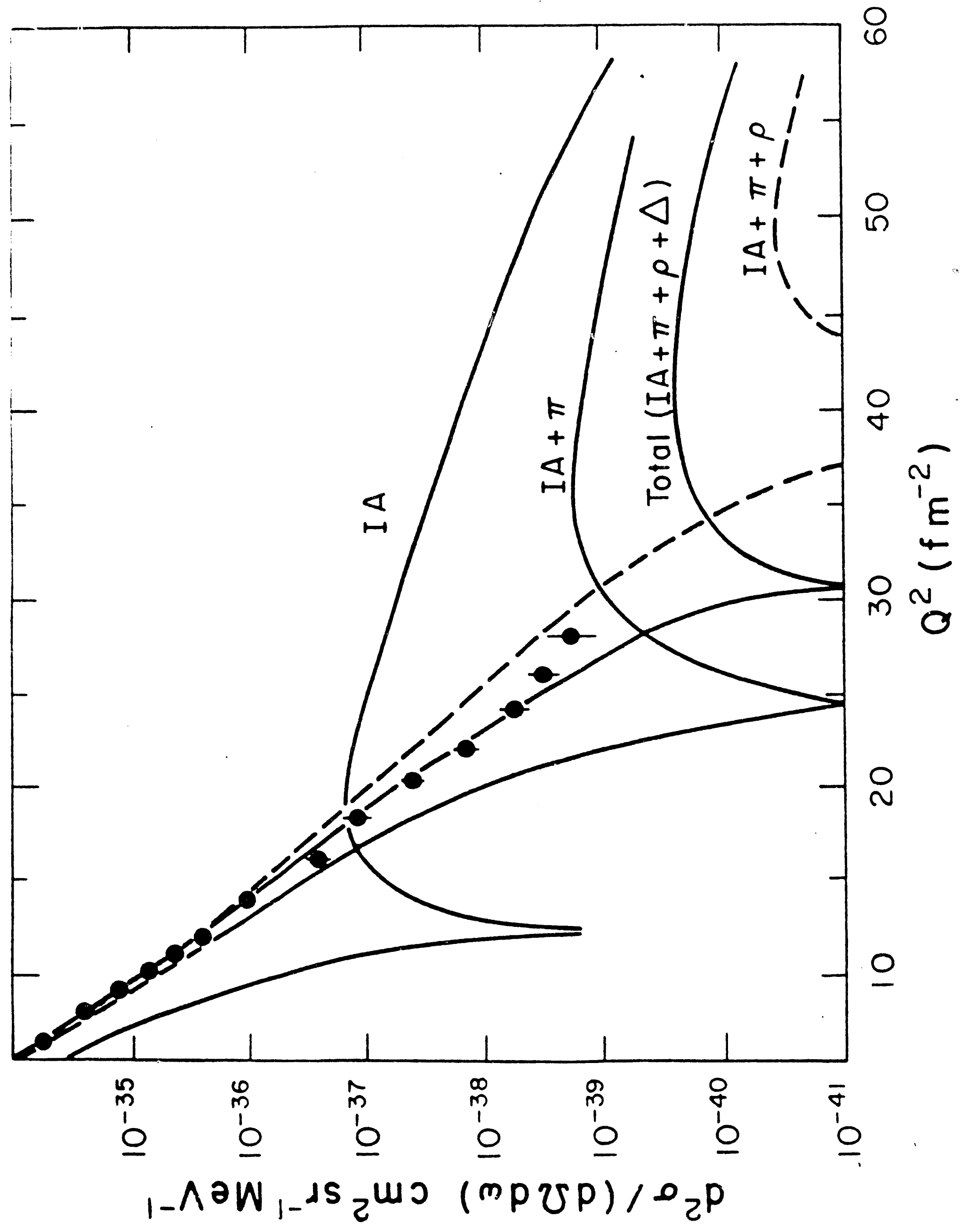




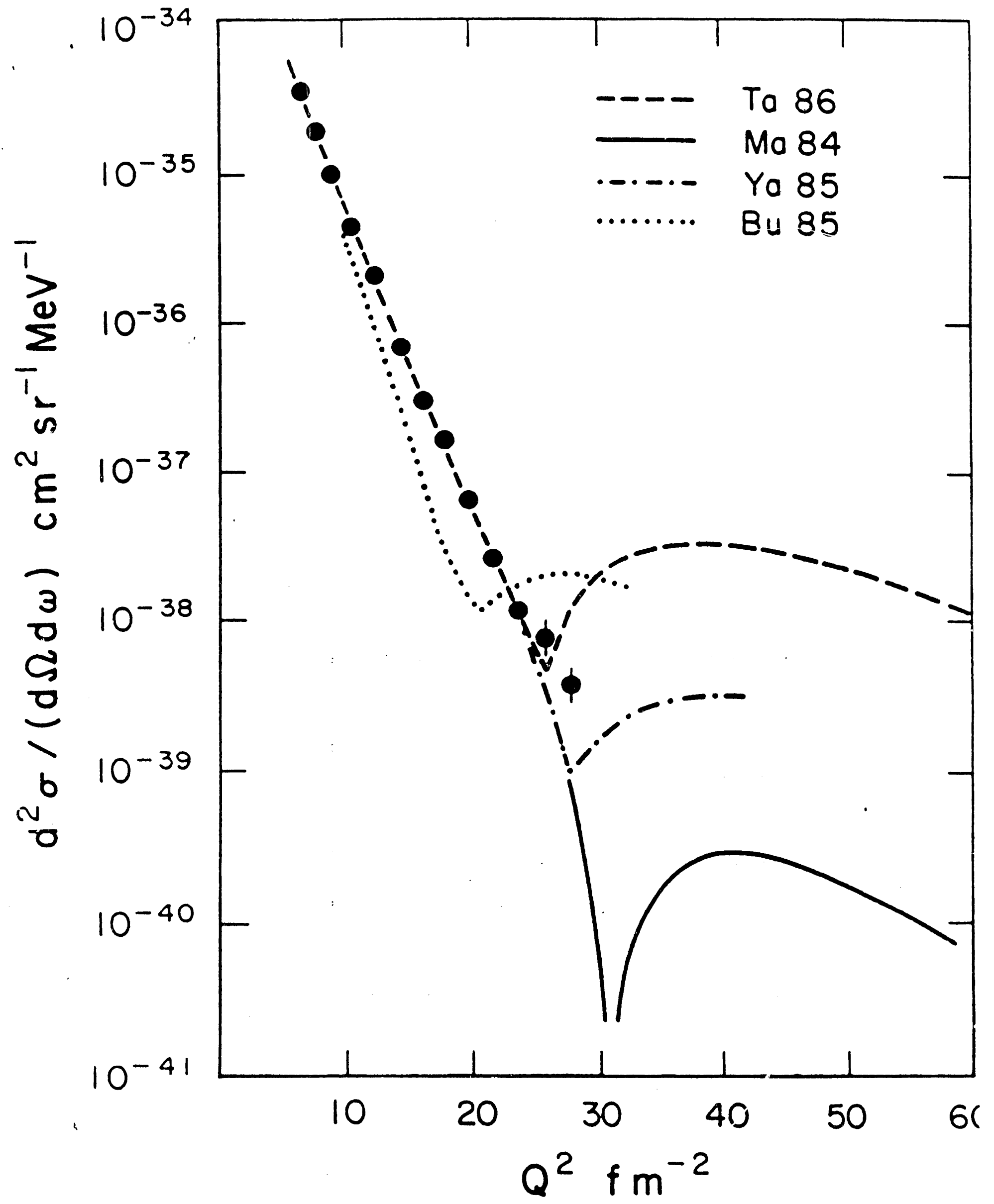


essential. Incident beam energies as high as $950 \mathrm{MeV}$ are required to obtain momentum transfers as high as $Q^{2}=45 \mathrm{~m}^{-2}$. Furthermore, a reasonable resolution must be obiained. It was realized that the necessary unique combination of energy, lumincsity, and resolution could only be obtained if the ELSSY spectrometer detection system was improved upon. This reo,ired the building of a new transverse array drift chamber, which would better define the vertical angle of the electron trajectory so as to distinguish event electrons arriving at the correct angle from background electrons at other angles. It also required the use of lead-glass shower counters which will uniquely identffy electrons when they are accompanied by other particles in the detection system. At this time the drift chambers have been completed, bench tested, and dellvered to Bates. The lead-glass shower counters have been borrowed from the SLAC NPAS program. They were assembled and tested by the American University group and are ready to be installed.

In addition there are equipment items that have not been completed yet. Our Saclay collaborators are bullding a gas Cerenkov that is expected to be completed and delivered to Bates near the end of this year. This component of the detection system should further ald in the discrimination of background from true events by rejecting non-relativistic particles, such as negative pions, from completely relativistic electrons in this single arm experiment.

Another component necessary for this experiment to commence is a llquid deuterium target. The drawings of the target cell were completed before the summer by Baies engineers, but no action has been taken yet to construct the new target cell because of a long list of other commitments at Bates and a shortage of personnel and money. The helium refrigeration system for cooling the deuterium must be moved from the South Hall at Bates to the North Hall and reinstalled. This is a complicated process that required considerable help from the Bates engineering and technical support groups.

Considering the complexity of this experiment and the difficulty of the installation of the refrigeration system, the detection system, etc., it has been decided to not run this experiment during the current Bates running cycle through March of 1989. These complexities coupled with the lack of rellability in obtaining and maintaining the necessary high energy recirculated beam at, e.g., $950 \mathrm{MeV}$, led to the decision to postpone. Instead, the 
experimental equipment will be brought on line in two phases. First the detection system and sottware will be thoroughly tested in approved UMass experiments (see below), and then the target, refrigerator, etc., will be assembled for the deuteron experiment. We are now aiming to run t'is experiment in the fall 1980 running cycle.

b. Expesiment 82-11: Electroexcitation of Discrete Levels in ${ }^{12} \mathrm{C}$ and $13 \mathrm{C}$ at

\section{Hioh Momentum Transter}

At momentum transfers of $Q>3 \mathrm{fm}^{-1}$ the wavelength of the virtual photon exchanged in electron scattering is such that nuclear features as small as a few tenths of a femtometer are probed. Moreover, the electromagnetic interaction is relatively weak so that the microscopic depth of fleld includes the deep interior of the nucleus. Since the interaction is also well understood, high-momentum-transfer electron scattering is a valuable technique for searching for short-range nuclear interaction effects, such as meson exchange currents. The relative contributions of exchange currents are in fact expected to be greatest at high momentum transfers, and Indeed, such $\left(\theta, \theta^{\prime}\right)$ measurements in the deuteron and $A=3$ nuclei have provided clear evidence for exchange currents in nuclel, as was described for experiment 87-02.

The lack of corresponding evidence for exchange current effects in heavier nuclei remains as a difflculty in our fleld. Our measurements of the elastlc $\mathrm{M} 1$ form factor of ${ }^{13} \mathrm{C}$ showed a large enhancement at high momentum transfers, as has also been the case for lighter nuclel. However, our data defled explanation in terms of the usual nuclear structure models, even when exchange currents were included. (See Phys. Rev. C 36, 485 (1987)).

In order to search for additional clues about the origins of this discrepancy, the data on the M1 elastlc form factor of ${ }^{13} \mathrm{C}$ were extended to $Q=4.6 \mathrm{fm}^{-1}$. Data were also acquired on transverse exchatlons of other multipolarity: $M 4$ and E3 transitions in ${ }^{13} \mathrm{C}$ at $E_{X}=9.50$ and $3.09 \mathrm{MeV}$, and $M 1, E 2$, and $M 2$ excltations in ${ }^{12} \mathrm{C}$ at $15.11,16.11$, and 16.58 MeV. It is found that calculations in the lowest-order shell-model space fall to account for the measured $Q$-dependences of $E 1, M 1$, and $E 2$ form factors beyond $Q=2 \mathrm{fm}^{-1}$. On the other hand, form factor shapes observed for M2 and M4 multipoles are satisfactorily described to $Q=4 \mathrm{fm}^{-1}$ within the minimal 1 hw conflguration space. 
At this time it appears that the unexpectedly large M1 cross sections measured for ${ }^{13} \mathrm{C}$ at high-Q can be attributed to core-polarization effects. To probe this interpretation further, we are presently calculating the ${ }^{13} \mathrm{C}-12 \mathrm{C}$ ground state charge distribution difference. This difference reflects how the additional neutron polarizes the ${ }^{12} \mathrm{C}$ core. The analysis of the ${ }^{13} \mathrm{C}$ elastic data requires the subtraction of the ${ }^{13} \mathrm{C} M 1$ contribution. Although such an analysis was previously done by J. Heisenberg, at that time the knowledge of the M1 form was fragmentary. Our reanalysis of these data is now nearty complete. We have used the socalled Mainz model-independent analysis and several DWBA codes.

The Bates PAC approved 126 hours of beam time to extend measurements beyond $Q=4.6 \mathrm{fm}^{-1}$. Until the current running cycle, we have not sought running time because we knew that the ELSSY spectrometer detection system needed to be improved before a meaningful measurement could be made at higher momentum transfers. As was explained for Experiment 87-02, the present detection system does not adequately define the transverse angle of the electron. This results in many background events being recorded. This problem hopefully will be solved when the new transverse drift chamber built by UMass for Experiment 87-02 (high-Q deuteron threshold electrodisiniegration) is installed together with the lead-glass shower and the gas Cerenkov counters. This should make possible the measurement of cross sections below $10^{-40} \mathrm{~cm}^{2} / \mathrm{sr}$.

We have sought running time for this experiment during the current Bates running cycle. If it is to be run, it will be in February or March of 1989 as the UMass group has commitments for the NE-11 experiment at SLAC from January 9 through February 9. Running the solid target ${ }^{13} \mathrm{C}$ experiment should provide the opportunity to thoroughly check the new ELSSY detection system, plus the data acquisition software which is considerably more complicated than that required for the old system. After this is done, further preparations for the deuteron experiment can be made, such as the installation and testing of the reirigerator and the liquid deuterium target. This experiment on ${ }^{13} \mathrm{C}$ then can serve as a precursor to the running experiment 87-02 on the deuteron. 
c. Experiment 83-03: $10 \mathrm{~B}\left(\theta, \theta^{\prime}\right) \mathrm{M3}$

There are 43.2 hours still available for this experiment. The objective for these - remaining beam hours is to obtain one more high- $Q$ point for the $1.74 \mathrm{MeV} 10_{B} \mathrm{M} 3$ form factor in order to still better define the $1 p_{3 / 2}$ wave function. Low- $Q$ data acquired in November of 1987 at NIKHEF will also help to define the wave function. This solid target experiment will serve as a precursor to Experiment 87-02 on the deuteron, as will experiment 82-11 on ${ }^{13} \mathrm{C}$. This experimen: also relates to the $\left(\theta, \theta^{\prime} p\right)$ coincidence experiment carried out on $10_{B}$ at NIKHEF this past summer, as will be explained.

\section{Experiments at Bates: Data Acquisition Receutly Completed}

\section{a. Experiment 87-05: Elastic Magnetic Electron Scattering from ${ }^{41} \mathrm{Ca}$}

The form factors of nuclei having doubly-closed shells with an additional single particle provide especially detailed and quantitative evidence about nuclear independent particle motion. The magnetic form factor in particular consists of a sum of convection and magnetization current amplitudes, and provides a means of sensitively testing various model assumptions. One example that has received much attention is the elastic magnetic form factor of 17 o. In this case, there is one neutron in the $d_{5 / 2}$ orbital outside the doubly-closed shell ${ }^{16}$ O nucleus, and the elastic magnetic scattering involves the three multipoles, M1, M3, and M5. Recent work on 170 by Bertozzl's group at Bates has extended the effective momentum transfer range from 2.47 to $3.65 \mathrm{fm}^{-1}$. It was found that the data follow a Woods-Saxon shell-model calculation where the M5 multipole dominates the form factor. Furthermore, it was found that many-body effects, such as meson exchange currents or core polarization, which were expected to be important at higher $Q$, do not seem to play a role in the above momentum transfer range. However, the data are lower than the Woods-Saxon results in the $Q$ range from 0.7 to $1.8 \mathrm{fm}^{-1}$. In this range the $\mathrm{M} 3$ multipole component in the form factor is suppressed. If a deformed ${ }^{16} \mathrm{O}$ core is taken into account, presumably this suppression can be understood.

It is of great interest to acquire elastic magnetic form factor data on other doublyclosed shell plus one-particle nuclel. Unionunateiy 170 and ${ }^{209}{ }_{B i}$ are the oniy iwo such 
nuclei that are stable. Notwithstanding, however, an experimental effort was begun at Saclay to measure elastic magnetic scattering from radioactive ${ }^{41} \mathrm{Ca}$. This nucleus has a $7 / 2$ neutron beyond the doubly-closed shell core of ${ }^{40} \mathrm{Ca}$. Data were acquired in July of 1986 in the momentum transfer range from 1.8 to about $3.3 \mathrm{fm}^{-1}$ at a scattering angle of $155^{\circ}$. The results bear upon the Nolen-Schiffer coulomb energy anomaly. They were published in Phys. Rev. Lett. 61, 1465 (1988).

There was also good reason to acquire data in the momentum transfer region below that of the Saclay data. Orie assumption in extracting the valence nucleon rms radius is that the lower multipoles $M 1, M 3$, and $M 5$ do not make large contributions in the higher $Q$ range. However, any anomalous behavior of the lower multipoles in the region below $Q=1.7 \mathrm{fm}^{-1}$ might indlcate problems in the ms radius extraction from the higher $Q$ range. Furthermore, it is of interest to measure the lower multipoles in order to obtain information on core polarization and the distortion of the ${ }^{40} \mathrm{Ca}$ core.

At the Saclay scattering angle of $155^{\circ}$, however, it would be difficult to measure the lower multipole components of the form factor. The scattering from the nuclear charge would dominate that from the magnetization densitles below about $Q=1.7 \mathrm{~m}^{-1}$ for ${ }^{41} \mathrm{Ca}$. In order to observe the magnetic scattering it would be necessary to go to larger backward angles. Therefore, in March measurements were made in the momentum transfer range from 0.9 to $1.9 \mathrm{fm}^{-1}$ by $180^{\circ}$ electron scattering at Bates. For the lower $\mathrm{Q}$ data it was absolutely essentlal to measure at $180^{\circ}$ so that the scattering from the nuclear charge distribution was minimized.

Since only a small amount of this rare radloactive isotope was avallable, the target was in the form of $\mathrm{CaCO}_{3}$ about the size of an aspirin tablet. Thus special methods of beam monitoring were used to keep the well-focussed beam at the same position on this very small target. For this purpose we built in our UMass shop a split-foll secondary-emission monitor (SEM) which was mounted directly on the target frame. The center of the target could be adjusted during mounting to coincide with the center of the SEM. This eliminated errors in attempting to align the SEM Independently of the target. The folls of the SEM were made of 5 mil graphite so that any electron striking them after passing through the iargei would lose 
valence orbits, and the relative differences in subshell occupation numbers with neighboring nuclel. A collaboratlve paper with Mainz and Amsterdam is still unfinished.

\section{b. Experiment $76-16: 12 C\left(\theta, \theta^{\prime}\right)$ at $180^{\circ}$}

The data acquisition for this experiment was completed in 1979. The data from this experiment as well as data acquired in tune-up runs for other experiments using ${ }^{12} \mathrm{C}$ as a callbration target have been used in five papers that have been published. There is still one more paper to be written in collaboration with J. Flanz of MIT-Bates on the $3^{-} 9.6 \mathrm{meV}$ excitation and other discrete traisverse electric excttations in ${ }^{12} \mathrm{C}$.

\section{c. Experiment 80-4: Transverse Electron Scattering from ${ }^{14} \mathrm{C}$}

A Ph.D. thesis has been written by M.A. Plum on the results of this experiment. A paper describing the $\mathrm{j}^{\pi}=4^{-}$states at $11.7,17.3$, and $24.3 \mathrm{MeV}$ and their isoscalar and isovector amplitudes of the spin-transition densities has been published. A second paper pertaining to other states in ${ }^{14} \mathrm{C}$ which were excited by $180^{\circ}$ electron scattering is nearing the final draft stage. It is being composed together with R.A. Lindgren who is now at the University of Virginla.

\section{d. Experiment 84-30: Elastle Magnetle Scadering from $89 y$}

This experiment was married to experiment 84-22 of the University of New Hampshire. The Bates PAC allotted 140 beam hours to it. Measurements of the magnetlc elastic cross sections for ${ }^{89} Y\left(\mathrm{~J}^{\pi}=1 / 2^{-}\right)$in the momentum transfer range from 0.8 to $2.8 \mathrm{fm}^{-1}$ were finished in January of 1987 . Inelastlc data obtained simultaneously will also provide information on the transverse components of the excited state form factors.

\section{Experiments Complete and the Results Publlshed}

Since the last progress report, the following experiments have been completely analyzed, and papers witten and published or submitted for publication. The reader is referred to the list of publications in part IV. 


\section{a. Experiments 82-02 and 83-07: Transverse and Longitudinal Electron Scattering from ${ }^{7}$}

These are collaborative experiments with the Tel Aviv group and with S. Kowalskl of MIT. A paper has been submitted for publication in Physics Letters. The abstract of this paper is as follows:

"Longitudinal and transverse electromagnetic form factors of the ${ }^{\mathrm{Li}}$ ground-state doublet (the ground state with $\mathrm{J}^{\pi}=3 / 2^{-}$and the first excited state at $E_{X}=478 \mathrm{keV}$ with $\mathrm{J}^{\pi}=1 / 2^{-}$) were measured by electron scattering up to momentum transfers of 4.2 and $4.5 \mathrm{fm}^{-1}$. respectively. The transverse form factnre show no structures in the high momentum transfer region which could be unambiguously identifled as signatures of meson-exchange currents; however, they lie above existing calculations which do not include such contributions. The longitudinal elastic form factor has a second maximum as does the C2 form factor of the 478 keV first exclted state."

\section{b. Experiment 83-03: The M3 Form Factors of the Boron lsotepes}

Transverse form factors have been measured for elastlc electron scattering from ${ }^{10_{B}}$ and ${ }^{11} \mathrm{~B}$, and for the electroexcitation of the $1.74 \mathrm{MeV}\left(\mathrm{J} \pi=0^{+}, T=1\right)$ and $5.17 \mathrm{MeV}\left(2^{+}, 1\right)$ levels in ${ }^{10} \mathrm{~B}$. A paper was published in Phys. Rev. Letters under the ttlle: "Determination of Single Nucleon Wave Functions by Transverse Electron Scattering." As was mentioned in a previous section, 43.2 hours of beam time still remain for a high-Q point to be measured.

\section{Experiments Planned}

If time permits, the UMass group will submit two proposals to the Bates PAC by the October 21, 1988 deadline.

\section{a. eluster-structures in $6 \mathrm{LI}$}

The Russian physicist R.A. Eramzhyan has pointed out to the principal investigator that the "bumps" in the high-Q longitudinal form factors of ${ }^{7} U$ for both the ground state and the first excited state can be accounted for by clustering in the nucleus. (See II.4.a. on Experiments 82-02 and 83-07.) It would be Interesting to carry measurements on another light nucleus to higher- $Q$ in order to check on clustering. 
The IJMass group has just completed building drift chambers that will permit such highQ measurements with a high discrimination against background for the low cross sections to be measured. The nucleus ${ }^{6} L$ is a good candidate, and furthermore, theorist $D$. Lehman at George Washington University has made cluster model calculations for ${ }^{6} \mathrm{LI}$. UMass made an alcohol-cooled target holder for the ${ }^{7} U$ experiment which also would be necessary here. Thus we would be prepared to expeditiously undertake studies of $6 \mathrm{LI}$.

\section{b. Qut of Plane Measurements of $d\left(\theta, \theta^{\prime} p\right)$ Coincidence Cross Sections Near} Threshold

This proposal would be made in collaboration with our colleagues in Japan: T. Tamae of Tohoku, A. Hotta of Shizuoka, and A. Matsuyama of Shizuoka. Tadaki Tamae of Tohoku would be the primary spokesman. This experiment would utlize the MEPS and OHIPS spectrometers at Bates to measure the $\phi$ dependence of electrons in coincidence with protons without the necessity of building new appa:atus such as that proposed by Papanicolas. For measurements near threshold, the ejected protons are emitted within a small cone along the momentum transfer direction for the small $E_{n p}$ and large $Q^{2}=8.7 \mathrm{~mm}^{-2}$ planned for the experiment. Scattered electrons would be detected in OHIPS and ejected protons in MEPS. The $\sigma_{L}+\sigma_{T}$ cross section and the $\sigma_{L T}$ interference cross section would be measured as a function of $\theta_{p}^{\mathrm{cm}}$. This experiment would provide more information of mesonexchange currents, iscbar configurations, form tactors, etc., than could be obtained in a single arm experiment.

The proposal will be fleshed-out by Monte-Carto calculations when Tamae retums to UMass in late November. The presentation to be given at the PAC on December 8 and 9 should be considerably more detalled than the proposal.

This work would be part of the UMass-Japan collaboration initlated by Professor Hotta of Shizuoka and the principal investigator.

B. Experiments at the llinols Microtren

A small, but an important part of our electron scattering program is being pursued by P. Mlskimen at the University of Massachusetts microtron. It is based on the use of 
coincidence $\left(\theta, \theta^{\prime} X\right)$ reactions. This choice is dictated by the principal advantage of the Illinols facility: the continuous nature of lts electron beam.

It is important that the UMass group acquires experience in the use of continuous duty factor facilities by performing coincidence experiments. Clearly such experiments will be standard fare in the 1990's when CEBAF and other facilities become operational. By becoming acquainted with the techniques and the theory of coincidence experiments, the UMass group should be ready to contribute to the electromagnetic interaction nuclear physics of the next decade.

During the past two years Professor Miskimen has developed detector systems capable of handling many of the particles emitted in nuclear de-excitation. These include several types of neutron detectors. Glant multipole resonances in heavy and medlum-heavy nuclei decay almost exclusively by neutron emission. Most of the decay is statistical, although a significant component (10-15\%) is found to be semi-direct or pre-equilibrium in character. For these cases the decay neutron was used only to "tag" the scattered electron as being associated with an inelastic event, as no energy of angular information is extracted from the neuiron branch. Such a measurement was completed on ${ }^{116} \mathrm{Sn}$ in March of 1987. The region of nuclear excitation studled by the $\left(\theta, \theta^{\prime} n\right)$ reaction was between $10 \mathrm{MeV}$ and $20 \mathrm{MeV}$, so as to cover the region of the electric monopole, dipole, and quadrupole giant resonances (EO, E1, and E2). For the kinematics of this experiment all three giant resonances are expected to make significant contributions to the cross section. In order to separate the strengths of these overlapping resonances, data were taken at three momentum transfers:

$q_{e f f}=0.37 \mathrm{fm}^{-1}, 0.45 \mathrm{~mm}^{-1}$, and $0.56 \mathrm{fmin}$ two separate runs. These data were combined with existing $(\gamma, n)$ data in a DWBA analysis to separate E1 from E0/E2 strength.

A first draft of a paper is nearly complete and will soon be sent to Miskimen's collaborators.

Other work done at IIIInois is related to CEBAF activities and is given in the sectlon on CEBAF. 


\section{Experiments at the Amsterdam NIKHEF-K Accelerater}

\section{Experiments Becently Completed}

An $\left(\theta, \theta^{\prime} p\right)$ coincidence experiment on ${ }^{10} \mathrm{~B}$ was done in June and July of 1988 in collaboration with C.W. de Jager, L. Lapikas, and $H$. de Vries of NIKHEF-K. The NIKHEF dual dispersion-matching spectrometer is the only one in the world having adequate energy resolution in both the electron and proton arms to carry out the measurement.

As a precursor to the coincidence experiment we completed the single-arm measurement of the form factor for the electroexcitation of the $1.74 \mathrm{MeV}$ state in ${ }^{10} \mathrm{~B}$ in the momentum transfer range from 0.5 to $2.0 \mathrm{fm}^{-1}$. These data will supplement more accurate higher $Q$ data obtained at Bates in experiment $83-03$, as well as low- $Q$ Saskatoon data of rather poor (20\%) accuracy. This is a pure $M 3$ transition since the ground state has spin and parity $3^{+}$and the $1.74 \mathrm{MeV}$ state has $0^{+}$. The objectlve is to accurately determine the $P_{3 / 2}$ wave function as ths $M 3$ transition can be interpreted in terms of $\left(p_{3 / 2}\right)^{6}$ recoupling. A welldetermined wave function would greatly aid in disentangling the ingredients entering into the interpretation of the $\left(\theta, \theta^{\prime} p\right)$ reaction, l.e.. the initial single-particle momentum distribution, the final state wave function, and the electron-proton interaction in the final state. For this single arm measurement 72 hours of beam time were used. The data acquired have not been analyzed yet.

It is hoped that these experiments will serve as benchmarks for the evaluation of final state interactions, since there should be a good understanding of the other ingredients entering Into the $\left(\theta, \theta^{\prime} p\right)$ reaction. However, one disadvantage of the $M 3$ transition is that it is of isovector character. This alows exchange currents to play a non-negligible role. Professor Dubach has calculated exchange current contributions to form form factors for many cases, and will assess exchange current contributions to the $\left(\theta, \theta^{\prime} p\right)$ cross sections.

\section{Experiments Completed and Published}

A paper was published in Physics Letters 205, 22 (1988) on the ground state. Since the ${ }^{15} \mathrm{~N}$ ground state has spin and parity $\mathrm{J}^{\pi}=1 / 2$, the ground state elastic form factor has only Coulomb monopole (CO) and magnetic dipole (M1) components. The CO form factor wos 
obtained from the NIKHEF data in the momentum transfer range from 0.4 to $3.2 \mathrm{fm}^{-1}$ after subtracting the M1 $180^{\circ}$ UMass results from Bates (Phys. Rev. C 28, 513 (1983)). Shell model calculations describing the $\mathrm{CO}$ form factor were performed by the Utrecht theory group. A closed ${ }^{4} \mathrm{He}$ core was not assumed, and excitations up to $2 \mathrm{h \omega}$ were included. These Calculations describe the CO data well, except for the helght of the second diffraction maximum. A ohw model space is also able to describe the form factor, but leads to a harmonic oscillator length parameter $b=1.67 \mathrm{~m}$ instead of $1.77 \mathrm{fm}$. Thus the CO data alone do not discriminate between the use of the different model spaces..

Charge density differences, as first pioneered by UMass as described in Phys. Lett. 358, 217 (1971), are more discriminating. There is considerable disagreement when the experimental difference is compared to a simple single $1 p_{1 / 2}$ hole description of $15 N$, which should represent the differance between the charge densities of 160 and ${ }^{15} \mathrm{~N}$. There is much better agreement with the $2 h \omega$ shell-model charge density difference. This shows that the description of the ground state of ${ }^{15} \mathrm{~N}$ as a $1 \mathrm{p}_{1 / 2}$ proton hole coupled to the 160 core is too simple, and that $2 h \omega$ configurations play a considerable role.

\section{Experimenis at NIKHEF: Data Analysis Completed}

a. A second draft of a paper describing longitudinal scattering from odd parity states in ${ }^{15} \mathrm{~N}$ has been completed. Probably one or two more drafts will be necessary betore a paper can be submitted for publication. Comparison of the data to $2 h w$ shell-model calculations of Dubach and Glaudeman's will be made.

b. A simllar paper is projected for the ${ }^{15} \mathrm{~N}$ positive partity levels. However, the main collaborator in this work, R.P. Singhal of Glasgow, is now involved in other experimental work so that it may be some time before a paper materializes.

\section{Experiments Planned at CEBAF}

Although it will be at least 5 years before beam is obtained at CEBAF, letters of intent were submitted in October of 1987, and updates to those first round of letters are due November 29, 1988. The University of Massachusetts submitted three letters of intent, in each of which it was requested to include a statement of commitment. Abstracts of these are given below as sections 1 through 4. 
reaction are essential in order to understand hypernuclear excitation through kaon photoproduction or electroproduction.

\section{Commitment of the Collaboraters}

At this time the University of Massachusetts group plans to focus its efforts entirely on the use of the Large Acceptance Spectrometer as each of its three letters of intent indicate. It is recognized that complicated reactions can be observed by using the LAS that cannot be studied with separate smaller spectrometers. Furthermore, the LAS should give a more efficient use of beam time as data will be collected over a broad range of experimental parameters.

We will commit our efforts to the construction of LAS hardware, such as drift chambers or shower counters. Our group has experience along these lines at both Bates and SLAC. Currently we are involved in constructing a new detector array for the $900 \mathrm{MeV}$ ELSSY spectrometer at Bates. We are also involved in the design of the detection system for the PEGASYS project at SLAC's PEP storage ring. If this project is funded, we probably will take responsibility for the drift chambers. Since one of our letters of intent bears upon the use of tagged photons, we would also expect to contribute to the construction of the photon tagging facillty.

Our faculty in experimental nuclear physics (R. Hicks, R. Miskimen, and G. Peterson) are now heavily committed to major experiments at Baterand SLAC, as well as to construction efforts, and in addition we hope to perform a coincidence experiment at NIKHEF. We realize that these expertments will span several years and that we are shorthanded if we are going to make contributions of substance at CEBAF. We are, therefore, trying to obtain another faculty position partially funded by the DOE, with the stipulation that the new faculty member would orlent most of his or her efforts toward CEBAF.

5. Measurements at the University of lllinols Pertaining to Projected Luminesties of the Large Acceptance Spectrometer (LAS) at CEBAF

The signal to noise ratio for a large detector such as LAS is proportional to $(1 / L)^{n-1}$ where $n$ is the number of particles in the final state and $L$ is the luminosity defined as the product of the number of electrons per second $N_{\theta}$ and the number of target nuclel per 
centimeter ${ }^{2}$. In turn $L$ is proportional to the product of beam current and target thickness. Thus the capabilities of LAS are strongly tied to the luminosity, which in turn is related to the background level. Since it is Intended to operate the LAS diff chambers close to the beam, a severe background from low energy electrons or photons could result in a greatly reduced luminosity. These backgrounds were estimated for various targets at CEBAF by using the electron-gamma shower code EGS-4 that was developed at Stantord. The results showed that a luminosity of at least $10^{33} \mathrm{~cm}^{-2} \mathrm{sec}^{-1}$ was possible with most targets. However, this is such an important quantity that an experimental check was merited. With this in mind, Baghael and Miskimen of UMass joined others for background tests of drift chambers at the University of Illinols In January and Aprll of 1988. Miskimen provided the drift chambers and had experience at the llinols $100 \%$ duty factor microtron.

Wire chambers were placed in a lead shieiding house through which the chambers directly viewed the beam through a variable magnetlc fleld. The background resulting from a target placed in the beam was studied as a function of target thickness, $Z$, angle, and beam energy. The results were compared to the EGS-4 calculations. It was found that low energy photons in the keV range could cause a substantlal background, but that they could be greatly reduced by thin lead and beryllium shielding around the beam pipe. The EGS-4 code needed some corrections. The ultimate result was that luminositles of the order of $10^{34} \mathrm{~cm}^{-2} \mathrm{sec}^{-1}$ should be possible - better than previously expected.

It should be noted that probably the first experiment that UMass would be involved with would be number 3 above, $\gamma+p \rightarrow K^{+}+\Lambda^{\circ}$, where real photons would first be used on the most background-free target, hydrogen. A large luminostty should be obtained for this experiment. 


\section{E. EXPERIMENTS AT SLAC}

\section{Experiment NE4: Electron Scattering from the Deuteron at Large}

\section{Momentum Transfers}

For this experiment two spectrometers were constructed and instrumented, one for backscattered electrons at $180^{\circ}$ and the other for recolling deuterons at $0^{\circ}$. In addition, a wellshielded moveable dump and a three magnet variable-angle chicane were required. The detector arrays included 14 planes of multt-wire proportional chambers, a gas Cerenkov counter, a segmented lead-glass counter, and planes of scintllators for timing purposes. The target system contained llquid deuterium and liquid hydrogen cells of lengths up to $40 \mathrm{~cm}$. The apparatus spread over much of the avallable space in End Station A.

As has been dlscussed in previous progress reports, data were taken during two periods: May-July 1985 and April-June 1986. The primary objective of this experiment was to increase the kinematic range of data on the magnetlc elastlc form factor of the decteron $B\left(Q^{2}\right)$. By extending the data out to $Q^{2}=2.77\left(Q_{\theta} V / c\right)^{2}$, it was shown that there was a diffraction minimum in $B\left(Q^{2}\right)$ near $Q^{2}=2\left(G_{\theta V} / c\right)^{2}$. The cross sections encountered at these high momentum transfers were extremely small, e.g., do/d $\Omega=(2.2 \pm 1.2) \times 10^{-41} \mathrm{~cm}^{2} / \mathrm{sr}$ at

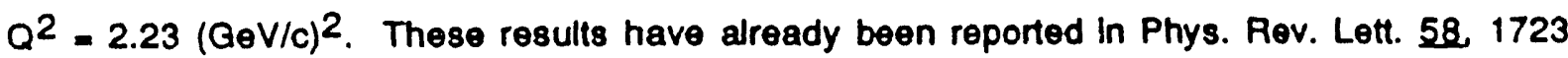
(1987).

During both the 1985 and 1986 experimental runs, Inelastic data were taken from threshold through the quasielastic region. These data were used to search for deviations from $y$-scaling, which would indicate the contribution of reaction mechanisms other than the simple impulse approximation. The data are in reasonable agreement with nonrelativistic models that include final-state interactions and meson exchange currents. The scaling function $F(y)$ derived from these data is generally in agreement with $F(y)$ obtained from forward angle data at the same $Q^{2}$. Values of $G_{M}^{n}$ determined from the data near the top of the quasielastlc peaks are in 
good agreement with results from previous experiments. These results were published in Phys. Rev. Letters 61,806 (1988).

One additional plece of Information is being extracted from this very large and moderately expensive experiment. UMass student Marc Frodyma is examining the breakup threshold region near 2.2 MeV excitation. Even through the experimental resolution varied between 12 and $20 \mathrm{MeV}$, it has been possible by judicious unfolding to obtain information about. the threshold reglon. Essentially cross sections for $180^{\circ}$ inelastic electron scattering were determined near break-up threshold for incident beam energies of $0.734,0.886,1.103,1.202$, and $1.279 \mathrm{GeV}$, corresponding to $1.21 \leq \mathrm{Q} 2 \leq 2.77\left(\mathrm{GeV}_{\theta} \mathrm{c}\right)^{2}$. Calculations by Arenhövel whilch includes MEC and ljobar conflgurations, arid a quark-hadron calculation by Yamauchi are to be compared to the data. Resolution unfolded cross sections, averaged over several $E_{n p}$ ranges, lle between several predictions at these values of $Q^{2}$. The $W_{1}$ inelastic structure function has been extracted from the data, and togeiner with $W_{2}$ from previous forward angle data, were used to calculate the ratio, $R=\sigma_{L} / \sigma_{T}$.

2. Experiment E140: Measurement of the $x, Q^{2}$, and A-Dependence of $R=\sigma_{L} / \sigma_{T}$ Early measurements of the quantity $R=\sigma_{\mathrm{L}} / \sigma_{\mathrm{T}}$, the ratio of the longltudinal and transverse cross sections for deep inelastlc lepton scattering, gave $R=0$, a result which was interpreted as evidence for spin 1/2 constituents within the nucleon. More recent theoretical advances in the QCD description of inelastic lepton scattering merit a much better determination of $R$ than is presently avallable. For example, current models consider contributions to $R$ from target mass effects and higher-order QCD. According to QCD theories, $R$ is expected to fall like $Q^{-2}$ as the four-momentum transfer $Q$ is increased. It is also important to Investigate the dependence of $R$ on $x$, the Bjorken scallng parameter, and $A$, the mass of the target nucleus. Any A-dependence of $R$ would have a significant Impact upon the understanding of the EMC effect, and, in particular, may account for differences observed betweer muon and electron data at low $x$. Furthermore, precise data on $R$ would permit an improved determination of the poorly-known longltudinal cross section $\sigma_{L}$, an important 
quantity inasmuch as it provides information on the quark and antlquark momentum distributions inside the nucleus.

Results from this experiment have recently been published in Phys. Rev. Letters. The following abstracts from two of the papers illustrates what was leamed in the experiment.

In Phys. Rev. Lett. 6e, 2591 (1988):"We measured the differences in $\mathrm{R}=\sigma_{\mathrm{L}} / \sigma_{\mathrm{T}}$ and the cross-section $\sigma^{A} / \sigma^{D}$ in deep inelastic electron scattering from $D, F \theta$, and $A u$ nuctel in the kinematic range $0.2 \leq x \leq 0.5$ and $1 \leq Q^{2} \leq 5\left(G_{\theta V / C}\right)^{2}$. Our results for $R^{A}-R^{D}$ are consistent with zero for all $x$ and $Q^{2}$, incidating that possible contributions to $R$ from nuclear higher-twist effects and spin-0 constituents in nuclel are not different from those in nucleons. The European Muon Collaboration effect is reconfirmed, and the low-x data from all recent experiments, at all $Q^{2}$, are now in agreement."

In Phys. Rev. Lett. 61, 1061 (1988): "We report new results on a precision measurement of the ratio $R=\sigma_{L} / \sigma_{T}$ and the structure function $F_{2}$ for deep-inelastic electronnucleon scattering in the kinematic range $0.2 \leq x \leq 0.5$ and $1 \leq Q^{2} \leq 10(\mathrm{GeV} / \mathrm{c})^{2}$. Our results show, for the first time, a clear falloff of $R$ with increasing $Q^{2}$. Our $R$ and $F_{2}$ results are in good agreement with QCD predictions only when corrections for target-mass effects are Included."

\section{Experiment NE11: DetermInation of the Nucleon Charge and Magnetlc Form} Factors at $Q^{2}=1.4$ to $6(\mathrm{GeV} / \mathrm{C})^{2}$

An understanding of the electromagnetic structure of the neutron and proton has been a central goal of particle physlcs for more than three decades. The need for comprehensive data has become more urgent in light of recent theoretical attempts to calculate nucteon structure from first principles of QCD. In particular, there is only limited data on the electric form factors, particularty that of the neutron. Because the neutron carries no net charge, $G_{E}^{n}=0$ at $Q=0$ and has beell generally assumed to be negligibly small at higher momentum transfers. Recently, however, Garl and Krumplemann noted that the incomplete data may in fact be consistent with a sizeable magnitude of $Q_{E}^{n}$ at $Q^{2} \geq 3(G \theta V / c)^{2}$. Gari and Krumplemann 
supported this observation with semiphenomenological calculations in a vector dominance model, modified to accord with QCD scaling predictions at large $Q^{2}$.

We propose to separate the charge and magnetic form factors of the proton $Q_{E p}$ and $G_{M p}$ at momentum transfers $Q^{2}$ from 1.4 to $6(\mathrm{G \theta V} / \mathrm{c})^{2}$, and those of the neutron $G_{E n}$ and $G_{M n}$ at momentum transters from 1.4 to $4\left(G_{\theta} V / C\right)^{2}$. The SLAC 8 GeV spectrometer will be used to measure elastiv and inelastic scattering from the proton and quastelastic and inelastic scattering from the deuteron over an angular range from 12 to 75 degrees. In order to increase the angular range for the separation, the $1.6 \mathrm{GeV}$ spectrometer will be calibrated against the $8 \mathrm{GeV}$ spectrometer at low and moderate $O^{2}$ at 75 degrees, and used to take high $\mathrm{Q}^{2}$ data at 105 degrees. It will have two 10018 quadrupoles placed in front of it to increase ths solid angle to about twenty times that of the $8 \mathrm{GeV}$ spectrometer. Beam energies from 1.1 to 5.5 GeV at maximum current will be required from the NPI operating in regular mode, and 10.2 GeV required from the NPE operating in SLED mode.

Protessor Hicks and Postdoctoral Research Assoclate Sayed Rokni have been involved for the past year helping to prepare for this experiment. Since both the $8 \mathrm{GeV}$ and the 1.6 GeV spectrometers of End Station A are to be used, considerable effort had to be given to instrumentation.

This experiment is scheduled to run from January 9, 1989 to February 9, 1989. All of the UMass experimental faculty tunded by this grant, as well as Dr. Rokni, will participate.

\section{PEGASYS}

Because of the change of focus of the high-energy particle physics effort at SLAC, several of the intersection regions at the $16 \mathrm{GeV}$ PEP storage ring are avallable for other purposes. In 1985 SLAC Director Burton Richter encouraged nuclear physicists to evaluate the potential of the PEP faclity for nuclear physics studies. In February 1986 an internal gas source was tried at PEP. In January of 1987 a workshop on "Electronuclear Physics with Internal Targets" was held at SLAC. Since that time there have been regular meetings of a 
collaboration invoived in designing experiments and a detector for a PEP GAS TARGET SYSTEM (PEGASYS). A letter of intent was prepared for the January 1988 advisory meeting. Since that time the collaboration has been busy preparing a proposal which will first be submitted to SLAC management.

There have been major design changes since the 1987 letter of intent. The use of an old streamer chamber has been abandoned in favor of building a new magnet with much better fleld properties and an acceptance three times as large as before. The use of this Big Acceptance Dipole (BAD) in turn resulted in major changes in the detector conflgurations. Furthermore, It was determiner that the superconducting beam pipe, which excluded magnetic flux in the vicinity of the recirculating beam in the storage ring, could be replaced by a large iron plate across the midsection of the BAD magnet. Also the use of fleld clamps on the input to BAD have eliminated the need for the large air-core fleld bucking colls near the target. Finally it has been decided that a cold $\left(20^{\circ} \mathrm{K}\right)$ ctuster-jet target should replace the warm jet gas target of the previous design. Most of these design features have been used before at CERN by Professor Louis Dick of CERN and Milan. Professor Dick is now part of the PEGASYS collaboration. His success at using simllar equipmerit at CERN bodes well for the operation of the equipment of this project.

There are many important measurements that can be made with the PEGASYS detector which are too involved for an adequate treatment here. However; suffice it to say that PEQASYS should be capable of operating in a momentum and energy transfer region that cannot be reached at the projected CEBAF energy of $4 \mathrm{GeV}$. Furthermore the projected kinematic capabilities of PEGASYS are also not so high, as they would be at Fermilab energies, so that the interesting region of overlap between nucleon-meson descriptions and quark-gluon descriptions is unavallable for study.

During this past yoar Professor Hicks and Dr. Rokni made major contributions in the design of hardware and experiments for PEGASYS. A presentation will be made for NPAS PAC on Friday, January 13, 1989. 


\section{Theoretical Work}

\section{A. Nucleon-Nucleon Interactions}

In an ongoing project in collaboration with $W$. M. Kloet and R. R. Silbar, a relativistic model based on iterated one-pion exchange in an integral equation formalism has been applied to the $\mathrm{NN} \rightarrow \mathrm{NN \pi}$ reaction in a way that preserves and emphasizes the effects of two- and three-body unitarity. This year, we have published a comparison of our model calculations with the first data from LAMPF for polarization observables for the reaction $\mathrm{pp} \rightarrow \mathrm{pp} \pi^{0}$. We have also begun an attempt to use this model to understand the role of non-delta "background" channels in pion production for "delta kinematics" as discussed in, e.g., A. B. Wicklund et al., Phys. Rev. D34, 19 (1986). Finally, this model is being used to provide the strong interaction components necessary for a calculation of parity violation in elastic proton-proton scattering as will be discussed further below.

\section{B. Nuclear Structure}

Much of the work in nuclear structure this past year involved using our already existing large-basis shell model codes to calculate the structure needed for some of the theoretical applications discussed below and for comparison with some of the experimental measurements discussed above. This included calculations for $\mathrm{A}=7,10,14,15,26$, 28, and 30. As his senior honors project, a UMass widergraduate, J. Eisenberg, has successfully developed computer codes to calculate the unitary transformation needed to transform from the M-scheme basis which our large-basis shell model codes currently use to a "good$\mathrm{J}$ " representation within the same basis. He demonstrated that numerical accuracy will probably not be a problem in carrying out this transformation, even for very large basis calculations. However, some improvement in computation time must be realized if this approach is to prove viable. This transformation is the first step in streamlining and enlarging our shell model codes; with Mr. Eisenberg's contribution, we hope to complete this process in the coming year. 


\section{Electromagnetic Interactions}

This past year saw publication of a long paper (in collaboration with T. W. Donnelly and A. S. Raskin) discussing the applications of the $\left(e, e^{\prime} \gamma\right)$ reaction in studies of nuclear structure. Since the measurement of the $\gamma$ angular distribution is essentially equivalent to measuring the spin sub-state distribution of the nuclear state induced by electroexcitation, our formalism was constructed to explicitly parallel the formalism for electron scattering from polarized targets. In each case, one can use such measurements to separate the various multipole projections of the nuclear charge and current distributions. This provides a more detailed description of these distributions than can be obtained from single-arm inclusive electron scattering, where one measures only the longitudinal and transverse form factors (i.e., ine incoherent sum of the squares of the multipole projections of the charge and current distributions), and we conclude that the $\left(e, e^{\prime} \gamma\right)$ reaction can indeed provide a valuable and practical tool for studying nuclear structure. We have also recently published (in collaboration with T. W. Donnelly and I. Sick) an analysis of the $p(\vec{e}, e) p$ process. The (unpolarized) cross section for e-p scattering is dominated by the electromagnetic interaction (photon exchange) while the asymmetry for polarized electron scattering from protons is determined by an interference between electromagnetic (photon exchange) and weak neutral current ( $Z^{0}$ exchange) amplitudes. If the kinematics are chosen to minimize the contributions of the nuclear axial current, then measurement of both the unpolarized cross section and the polarization asymmetry provides two independent "views" of the vector current of the nucleon. This in turn allows for a decomposition of this current into isoscalar and isovector components and, from there, a "reconstruction" of the neutron electromagnetic charge form factor, $G_{E_{n}}$. It has, of course, been understood for some time that $G_{E_{n}}$ enters into the $p(\vec{e}, e) p$ process. Due to our present poor knowledge of the neutron electric form factor, attempts to use this process as a test of electroweak theories have chosen kinematics which deemphasize the sensitivity to $G_{E_{n}}$. We have simply taken the opposite approach, that the Standard model for the electroweak interaction has been shown to be sufficiently accurate so as to allow a determination of $G_{E_{n}}$ from the $p(\vec{e}, e) p$ process, at least for certain kinematic situations. We conclude that measurement of $G_{E_{n}}$ at 
intermediate momentum transfers at the $30-40 \%$ accuracy level is both possible and feasible at, e.g., CEBAF. We believe such a measurement can provide a useful complement to other more "conventional" methods for measuring $G_{E_{n}}$ using deuteron and A=3 targets. These methods involve nuclear physics uncertainties such as structure effects and final state interactions, while we have made entirely "orthogonal" assumptions, mainly involving the parity and isotopic purity of the nucleons. Thus agreement between the two approaches would provide a much greater confidence that we have finally accurately determined $G_{E_{n}}$. The above discussion of $p(\vec{e}, e) p$ emerged from a more general discussion of isospin dependences of parity-violating electron scattering, also carried out in collaboration with $\mathrm{T}$. W. Donnelly and I. Sick. We began with consideration of the case of elastic scattering from spin-0 $\mathrm{N}=\mathrm{Z}$ nuclei (such as the experiment on ${ }^{12} \mathrm{C}$ now underway at MIT-Bates). Analysis within the Standard model of electroweak.interactions shows that the polarization asymmetry for this process should be nuclear structure independent and proportional to $\sin ^{2} \theta_{W}$ where $\theta_{W}$ is the Weinberg angle. Thus, an accurate measurement of this asymmetry will provide a stringent test of the electroweak model and an accurate measurement of $\sin ^{2} \theta_{W}$. Such an analysis assumes, however, that the ground state is a pure isospin-0 state. We have investigated how isospin mixing between the $0^{+} 0$ ground state and excited $0^{+} 1$ states will destroy this analysis. We conclude that isospin mixing will introduce structure dependence into the expression for the polarization asymmetry. However, our worst-case estimates for ${ }^{12} \mathrm{C}$ show that this dependence can (somewhat fortuitously) be expected to be below the $1 \%$ level so that a significant measurement of $\sin ^{2} \theta_{W}$ is still possible. For other cases, such as ${ }^{28} \mathrm{Si}$, this is not the case, and isospin mixing effects can be well above the $1 \%$ level. We have extended this analysis to inelastic scattering and to $\mathrm{N} \neq \mathrm{Z}$ nuclei. In the latter case, we show that the polarization asymmetry depends directly on the neutron density in the nucleus and conclude that this may be a possible (though difficult) way to determine the neutron density. A paper detailing these results is in preparation. A graduate student, C. Arabadjis, has begun an investigation of the $\left(e, e^{\prime} p\right)$ reaction. The motivation for this work has come from the recent UMass-NIKHEF experiments on ${ }^{10} \mathrm{~B}$, and the short-range goal is to help provide an interpretation of that data. The longer-range goal is to include state-of-the-art calculations for all components which enter such calculations including 
shell-model wave functions, meson exchange currents, and final-state interactions. This is now intended to be the Ph.D. work of Mr. Arabadjis. Finally, we have begun to look at the process of hadronization following a high-energy electron-quark scattering. The struck quark can not emerge as a free particle from such a scattering but must dress itself on the way out, leaving behind only color-singlet hadrons. One of the interesting questions is how the struck quark, having left the immediate vicinity of its original nucleon will behave in the presence of the remaining A-1 nucleons in the target nucleus. Brodsky has suggested that this process should be A-independent (based on the idea of "color transparency"), at least for certain classes of events such as the production of single very energetic pions. We are examining the models and assumptions which go into this suggestion, and are also attempting to generalize to more complex events.

\section{Weak Interactions}

We have been using the nucleon-nucleon model of Kloet and Silbar (in collaboration with them and L. S. Kisslinger) to examine parity-violation in proton-proton scattering at intermediate energies. By sandwiching a weak potential between the strong $N N \rightarrow N N$ and $\mathrm{NN} \rightarrow \mathrm{N} \Delta$ scattering matrices from this model we can calculate the longitudinai polarization asymmetry, effectively using the rnodel to determine the distortions of the protons in a distorted-wave Born approximation approach. We have to date considered only onepion exchange as the source of the parity violation, but allow for weak transitions from $\mathrm{NN}$ to $\mathrm{N} \Delta$ states. Our calculations give a polarization asymmetry of about the same magnitude as that measured in a recent LAMPF experiment, but of opposite sign. We predict smaller but still significant asymmetries in the energy region of a present TRIUMF experiment. Small but irritating difficulties with these calculations have held up publication of our results for some time. However, we believe these difficulties have all been resolved. A preliminary draft of a paper is circulating among the coauthors, and should be submitted for publication shortly. In related work, a graduate student, G. Feldman, has been improving (in collaboration with B. Holstein) the weak potential to be used for such parity-violating $N N$ interactions. In particular, he has considered the exchange of $\rho$ and $\omega$ mesons in addition to pions; he has also included $\Delta-\Delta$-meson couplings. Use of this 
potential in the calculation of the $\mathrm{NN}$ polarization asymmetry requires modification of the procedures used in the calculations described above. These modifications are in progress. Completion of our work on the decay modes of hypernuclei was delayed by some questions about the way in which we had calculated some of the decay rates as reported in a number of conference proceedings. These questions have now been resolved (in our favor). We have decided to combine our results for nuclear matter and finite nuclei into a single paper, and that manuscript is nearly completed. The interesting physics in this problem is that the hypernuclear decay proceeds through the weak, strangeness-changing, two-body mechanism $\Lambda N \rightarrow N N$ rather than through the free-space decay mode of the lambda, $\Lambda \rightarrow$ $\mathrm{N} \pi$, at least for all but the lightest hypernuclei. This provides a rather unique look at both the parity-conserving and parity-violating components of the two-hadron weak interaction. Many models, based mainly on one-pion exchange, are capable of predicting the total decay rates. We have demonstrated, however, that the ratic of neutrons to protons in the final state is particularly sensitive to the form of the weak interaction and varies considerably from model to model. The measurement of this ratio is not very precise, but our model, in which the exchange of kaons plays a crucial role, is in reasonable agreenent with those experiments. More refined measurements of this ratio along with an experimental separation of the parity-conserving and parity-violating components will be most interesting.

\section{E. Relativistic Nuclear Physics}

In an ongoing collaboration between a post-doc in our group, G. A. Crawford, and R. N. Thaler various aspects of the relativistic two-body problem are being investigated. During the past year most of this work has centered on a new approximation technique for solutions of the Bethe-Salpeter enuation. This technique involves a factorization of the wave function into relative energy and relative momentum parts. This allows the BetheSalpeter equation to be reduced to a pair of coupled but simpler integral equations. A study of this approximation technique for a model problem has been carried out and the results submitted for publication. Detailed studies of this technique for a more realistic 
problems are planned for the fiture. Supercomputer access through a DOE program has been requested for work on this problem.

In related work, we are beginning a study to examine relativistic effects in nuclear shell models. As a "practice" problem, graduate student J. Gallant, has recently completed coding for numerically solving the Dirac equation to find the single-particle wave functions for use in a "relativistic shell model". The present intent of this work is to understand what others have done in making such models, and to assess the role of such relativistic effects in comparing shell model predictions with measured electron scattering form factors.

E. Other

Finally, G. Feldman has begun to explore models of the $(\pi, 2 \pi)$ reaction from nuclei. The emphasis here is to understand whether one can exploit this reaction to distinguish between different underlying models for the $\pi-\pi$ and $\pi \pi \mathrm{N}$ interactions. In related work, he is also examining whether pion interference effects (the Hanbury-Brown Twiss effect) can be exploited to gain rather unique information about the underlying pion production mechanisms. This work is only in a preliminary stage; a more definitive picture should emerge in the next few months.

\section{G. A Proposal by the UMass Particle Theory Group}

The UMass particle theory group (Professors J.F. Donoghue, E. Golowich, B.R. Holstein, and A.R. Swift) has submitted a proposal to the National Science Foundation for funding the salary of a postdoctoral research associate, who in collaboration with the above professors, would address a variety of research topics at the interface area between theoretical elementary particle physics and theoretical nuclear physics. There are already collaborations between physicists in these two areas of research at UMass. For example, Donoghue, Holstein, and Dubach have studied the weak non-mesonic decay of hypernuclei, Holstein and Dubach have 
examined the role of mesonic exchange currents in allowed nuclear beta decay, and Holstein together with Dubach and Silbar of Los Alamos are examining weak parity violation vertices in attempting to explain the Los Alamos polarized proton scattering asymmetry at $800 \mathrm{MeV}$.

It is not known whether this proposal from theoretical particle physics will be funded. However, if it is funded, it is apparent that the result could only strengthen the UMass effort in the class of physics to be pursued at CEBAF, and in the NPAS and PEGASYS programs at SLAC. 
- IV. Publications and Presentations by Members of the University of Massachusetts Medium-Energy Nuclear Physics Group in the Period July 1, 1987 to August 31,1988.

A. Journal Publications:

1. "Electroexcitation of Discrete Levels in ${ }^{12} \mathrm{C}$ and ${ }^{13} \mathrm{C}$ at High Momentum Transfers*, R.S. Hicks, R.L. Huttman, R.A. Lindgren, G.A. Peterson, M.A. Plum, and J. Button-Shafer, Phys. Rev. C 36, 485 (1987).

2. Transverse Isoscalar Excitations in $24 \mathrm{Mg}$ by $180^{\circ}$ Electron Scattering", A. Hotta, R.S. Hicks, R.L. Hutfman, G.A. Peterson, R.J. Peterson, and J.R. Shepard, Phys. Rev. C 36, 2212 (1987).

3. "A Search for Short-Lived Axions in an Electron-Beam Dump Experiment", E.M.Riordan, M.W. Krasny, K. Lang, P. de Barbaro, A. Bodek, S. Dasu, N. Varelas, X. Wang, R. Amold, D. Benton, P. Bosted, L. Chogher, A. Lung, S. Rock,Z. Szalata, B.W. Fillipone, R.C. Walker, B. Debebe, M. Frodyma, R.S. Hicks, G.A. Peterson, and R. Gearhart, Phys. Rev. Lett. 59, 755 (1987).

4. "Determination of Single-Nucleon Wave Functions by Transverse Electron Scattering", R.S. Hicks, J. Button-Shafer, B. Debebe, J. Dubach, A. Hotta, R.L. Huttiman, R.A. Lindgren, G.A. Peterson, R.P. Singhal, and C.W. de Jager, Phys. Rev. Lett. 60, 905 (1988).

5. "A Study of the Nuclear and Kinematic Dependence of $R=\sigma_{L} / \sigma_{7}$ in Deep Inelastic Electron Scattering", S. Dasu, P. de Barbaro, R.C. Walker, L.W. Whitlow, J. Alster, R. Amold, P. Bosted, D. Benton, A. Bodek, J. ButtonShafer, G. de Chambrier, L. Clogher, B. Debebe, F. Dietrich, B. Fillippone, R. Gearhart, H. Harada, R. Hlcks, J. Jourdan, M.W. Krasny, K. Lang, A. Lung, R. Milner, R. McKeown, A. Para, D. Potterveld, E.M. Rlordan, S.E. Rock, Z.M. Szalata, and K. Van Blbber, Nucl. Phys A478, 391c (1988).

6. The ${ }^{15} \mathrm{~N}$ Ground State Studied with Elastic Electron Scattering", J.W. de Vries, D. Doomhof, C.W. de Jager, R.P. Singhal, S. Salem, G.A. Peterson, and R.S. Hicks, Phys. Lett. B 205, 22 (1988).

7. Measurements of Transverse Quasielastic Electron Scattering from the Deuteron at High Momentum Transfer", R.G. Amold, D. Benton, P. Bosted, L. Clogher, G. DeChambrier, A.T. Katramaiou, J. Lambert, A. Lung, G.G. Petratos, A. Rahbar, S.E. Rock, Z.M. Szalata, B. Dobobo, M. Frodyma, R.S. Hicks, A. Hotta, G.A. Peterson, R.A. Gearhart, J. Alster, J. Lichtenstadt, F. Dietrich, and K. van Bibber, Phys. Rey. Lett. 61, 806 (1988).

8. "Measurement of the Difference in $R=\sigma_{L} / \sigma_{7}$ and $\sigma^{A} / \sigma^{D}$ in Deep-Inelastic $\theta$ $D, \theta-F \theta$, and $\theta-A u$ Scattering," S. Dasu, P. de Barbaro, A. Bodek, H. Harada, M.W. Krasny, K. Lang, E.M. Flordan, R. Amold, D. Benton, P. Bosted, L. Clogher, G. deChambrier; A. Lung, S.E. Rock, Z.M. Szalata, R.C. Walker, B.W: Fillppone, J. Jourdan, R. Milner, R. McKeown, D. Potterveld, A. Para, F. Dietrich, K. Van Blbber, J. Button-Shafer, B. Debebe, R.S. Hicks, G.A. Peterson, R. Gearhart, L.W. Whitlow, and J. Alster, Phys. Rev. Lett. 60, 2591 (1988).

9. The Reaction ( $\left.\theta, \theta^{\prime} \gamma\right)$ in Nuclel", T.W. Donnelly, A.S. Raskin, J. Dubach, Nuct. Phys. A474, 307 (1987). 
10. "Polarization Observables for the Reaction pp $\rightarrow p p \pi^{\circ}$ at $800 \mathrm{MeV}$ ", P.J. Riley, Mark Bachman, C.L. Hollas, K.H. McNaughton, S. XU, B.E. Bonner, O.B. van Dyck, J. McGill, M.W. McNaughton, J.C. Peng, J. Dubach, R.R. Silbar, and W.M. Kloet, Phys. Lett. 197B, 23 (1987).

11. Precision Measurement of $R=\sigma L / \sigma$ and $F_{2}$ in Deep Inelastic Electron Scattering", S. Dasu, P. de Barbaro, A. Bodek, H. Harada, M.W. Krasny, K. Lang, E.M. Rlordan, R. Arnold. D. Benton, P. Bosted, G. de Chambrier, L. Clogher, A. Lung, S.E. Rock, Z.M. Azalata, R.C. Walker, B.W. Fillippone, J. Jourdan, R. Milner, R. Mckeown, D. Potterveld, A. Para, F. Dielrich, K. Van Bibber, J. Button-Shater, B. Debebe, R.S. Hicks, J. Alster, L.W. Whitlow, and ,R. Gearheart, Phys. Rev. Lett. 61, 1061 (1988).

12. "Determination of the Neutron Electric Form Factor from $p(\vec{e}, \theta) p ", T . W$. Donnelly and J. Dubach, Phys. Rev. C.37, 2320 (1988)

B. Articles Accepted for Publication:

1. "Electroexcitation of "He in the near continuum", A. Hotta, J. Dubach, R.S. Hicks, R.L. Huttman, B. Parker, G.A.'Peterson, P.J. Ryan, and R.P. Singhal, To be published in Phys. Rev. C, October, 1988.

C. Articles Submitted for Publication:

1. "Relativistic two-body differential equatlons, integral equatlons, and boundary conditions", G.A. Crawford and R.M. Thaler, Submitted to Phys. Rev. C.

2. "Measurement of the $17 / 2$ valence neutron orbit radius in ${ }^{41} \mathrm{Ca}$ ", $\mathrm{S}$. Platchkov, A. Amroun, P. Bricault, P. Dreux, B. Frols, C.D. Goodman, D. Goutte, P. deWitt Huberts, J. Martino, X.H. Phan, G.A. Peterson, S. Raman, and I Sick, Submitted to Phys. Rev. Lett.

D. Articles Soon to be Submitted for Publication:

1. "Weak Nun-Mesonic Decay of Hypermuclei", L. de la Torre, J.F. Donoghue, J. Dubach, B.R. Holstein, and M. Kimura, to be submited to Annals of Physics.

2. "Isospin Dependences in Partty-Vlolating Electron Scattering", T.W. Donnelly, J. Dubach, and I. Sick, to be submitted to Phys. Rev. C.

3. $180^{\circ}$ Electron Scattering from ${ }^{14} \mathrm{C}$, M.A. Plum, R.A. Lindgren, J. Dubach, R.S. Hicks, R.L. Hutfman, B. Parker, G.A. Peterson, J. Alster, J. Lichtenstadt, M.A. Moinester, and H. Baer, to be submitted to Phys. Rev. C.

4. Longltudinal and Transverse Form Factors of ${ }^{7} \cup$ Measured at High Momentum Transfer", J. Lichtenstadt, J. Alster, M.A. Molnester, J. Dubach, R.S. Hicks, G.A. Peterson, and S. Kowalski, to be submitted to Phys. Rev. Lett.

5. "Long-Range Contribution to the Parity-Violating Asymmetry in pp Scattering", R.R. Silbar, W.M. Kloet, L.S. Kisslinger, and J. Dubach, to be submitted to Phys. Rev. C. 
E. Articles in Preparation:

1. "Inelastlc Electron Scattering from 15N", J.W. de Vries, C.W. de Jager, R.P. Singhal, S. Salem, G.A. Peterson, and R.S. Hicks. Being prepared for Phys. Rev. C.

2. "Octupole Transitions in 1p Shell Nuclei", S.A. Salem, R.P. Singhal, J.W. de Vries, C.W. de Jager, R.S. Hicks, G.A. Peterson, and D.J. Millener, Being prepared for Phys. Rev. C.

3. "Sell-Consistent Retardation in a Three-Dimensional Relativistic Equation", G.A. Crawtord and R.M. Thaler.

F. Publlshed invited Conference Talks: None

G. Invited Talks to be Published In Conference or Workshop Proceedings:

1. Transverse Inelastic Electron Scattering from the Deuteron on High Momentum Transfers", P.E. Bosted, R.G. Amold, D. Benton, L. Clogher. G. DeChambrier, A.T. Katramatou, J. Lambert, A. Lung, G.G. Petratos, A. Rahbar, S.E. Rock, Z.M. Szalata, B. Debebe, M. Frodyma, R.S. Hicks, A. Hotta, G.A. Peterson, R.A. Gearhart, J. Alster, J. Lichtenstadt, F. Dietrich, and $K$. van Blbber, Presented at 3rd Conference on the Intersections between Particle and Nuctear Physlcs, May 14-19, 1988, Rockport, Maine.

H. Unpublished Invited Talks at Conferences: None.

I. Contrlbuted Talks at Meetings or Conferences:

1. "Stretched M6 Form Factor in ${ }^{28} \mathrm{Si}$ at High Momentum Transter", H.M. Farkhondeh, R. Undgren, S. Auffret, T.S. Deng, J. Dubach, R. Hicks, R. Huttman, M. Leuschner, G.A. Peterson, M. Plum, P. Ryan, and R. Singhal, Bull. Am. Phys. Soc. 32, 1574 (1988).

2. "Factorized Approximations to Solutions of the Bethe-Salpeter Equation", G.A. Crawtord, Bull. Am. Phys. Soc. 33, 989 (1988).

3. 'A Precision Measurement of the Proton Electric Form Factor, $G_{E^{\prime}}^{P}$ in o-p Elastic Scattering", R.C. Walker, B.W. Fillppone, J. Jourdan, R. Milner, R. Mckeown, D. Potterveld, R. Amold, D. Benton, P. Bosted, G. DeChambrier, L. Clogher, A. Lung, S.E. Rock, Z.M. Szalata, A. Para, F. Dietrich, K. Van Bibber, J. Button-Shafer, B. Debebe, R.S. Hicks, S. Dasu, P. de Barbaro, A. Bodek, H. Harada, M.W. rasney, K. Lang, E.M. Rlordan, J. Alster, L.W. Whitlow, and R. Goarhart, Bull. Am. Phys. Soc. 33, 1098 (1988).

4. "Recent Searches for Short-Lived Pseudoscalar Bosons in Electron BeamDump Experiments", M.W. Krasny, R. Amold, P. de Barbaro, D. Benton, J.D. Bjorken, A. Bodek, P. Bosted, J. Button-Shafer, L. Clogher, M. Crisler, S. Dasu, B. Debebe, B. Fillppone, M. Frodyma, R. Gearhar, R.S. Hicks, J. Lambert, K. Lang, A. Lung, A. Para, G.A. Peterson, E.M. Rlordan, S. Rock, Z. Szalata, N. Varelas, R.C. Walker, and X. Wang, Presented by M.W. Krasny at the Europhysics Conf., Uppsala 1987. 
5. "Results from a precision Measurement of the $x, Q^{2}$ and Nuclear Dependence of $R=\sigma_{L} / \sigma^{*}$, S. Dasu, P. de Barbaro, R.C. Walker, L.W. Whittow, J. Alster, R. Amold, D. Benton, A. Bodek, P. Bosted, G. de Chambrier, L. Clogher, B. Debebe, F. Dietrich, B. Fillippone, R. Gearhart, H. Harada, R. Hlcks, J. Jourdan, M.W. Krasny, K. Lang, A. Lung, R. Milner, R. McKeown, A. Para, D. Potterveld, E.M. Rlordan, S.E. Rock, J. Shater, Z.M. Szalata, and K. Van Blbber, Presented by S. Dasu at the Europhysics Conference, Uppsala 1987.

6. Transverse Inelastic Electron Scattering from the Deuternn at High Momentum Transfers", P.E. Bosted, R.G. Amold, D. Bentc,i, L. Clogher, G. DeChambrier, A.T. Katramatou, J. Lambert, A. Lung, G.G. Petratos, A. Rahbar, S.E. Rock, Z.M. Szalata, B. Debebe, M. Frodyma, R.S. Hicks, A. Hotta, G.A. Peterson, R.A. Gearhart, J. Alster, J. Lichtenstadt, F. Dletrich, and $K$. van Blbber, presented at 3rd Conference on the Intersections between Particle and Nuclear Physics, May 14-19, 1988, Rockport, Maine.

7. "Precision Messurement of $R=\sigma_{L} / \sigma_{T}$ and $F_{2}$ in Deep Inelastic Electron Scattering on Deuterlum and Iron Targets", P. Bosted, S. Dasu, R.C. Walker, L.W. Whitlow, J. Alster, R. Amold, P. de Barbaro, D. Benton, A. Bodek, J. Button-Shafer, $Q$. deChambrier, L. Clogher, B. Debebe, F. Dietrich, B. Filippone, R. Gearhart, H. Harada, R. Hicks, J. Jourdan, M.W. Krasny, K. Lang, A. Lung, R. Milner, R. McKeown, A. Para, D. Potterveld, E.M. Rlordan, S. Rock, Z.M. Szalata, and K. Van Blbber, presented at 3rd Conference on the Intersections between Particle and Nuclear Physics, May 14-19, 1988, Rockport, Maine.

\section{J. Conference and Workshop Participation:}

CEBAF Summer Workshop, June 1987 (J. Dubach, R.A. Miskimen, and G.A. Peterson)

CEBAF Summer Workshop, June 1988 (H. Baghael, S. Rokni, and J. Dubach)

Gordon Conference on Photonuclear Reactions, August 8-12, 1988 (H. Baghael, J. Dubach, R.A. Miskimen, and G.A. Peterson)

PEGASYS Summer Workshop at SLAC, July 1988 (R.S. Hicks, and S. Rokni)

K. Seminars or Colloqula:

J. Dubach: University of New Hampshire, April, 1988.

J. Dubach: MIT-Bates, April, 1988.

G.A. Peterson: Saclay, France, November, 1987.

H. Baghael: R.P.I., May, 1988.

G.A. Crawtord: Ohlo State, April, 1988.

L. Publications by Members of the UMass Nuclear Physics Involving Work Primarliy Done Elsewhere:

1. "Isobaric-Analog-State Transitions in Plon Charge-Exchange Reactions Above the $\Delta(1232)$ Resonance", S.H. Beknil H.W. Baer, A.G. Bergmann, J.D. Bowman, F. Irom, M.J. Leitch, C.J. Seftor, J. Alster, E. Plasetzky, B.L. Clausen, R.A. Loveman, R.J. Peterson, J.L. Ullman, J.R. Comford, J.N. Knudson and U. Sennhauser, Phys. Lett. B 202, 35 (1988). 
2. "Missing-Energy Dependence of the Separated Response Functions for the Reaction ${ }^{12} \mathrm{C}\left(\theta, e^{\prime} p\right)^{*}$, P.E. Ulmer, H. Baghael W. Bertozzl, K.I. Blomquist, J.M. Finn, C.E. Hyde-Wright, N. Kalantar-Nayestanakl, S. Kowalskl, R.W. Lourle, I Nelson, W.W. Sapp, C.P. Sargent, L. Weinstein, B.H. Cottman, P.K. Teng, E.J. Winhold, M. Yamazaki, J.R. Calarco, F.W. Hersman, J.J. Kelly, M.E. Schulze, and G. Audt, Phys. Rev. Lett. 59, 2259 (1988).

3. Transition Densities in ${ }^{30}$ SI Studied by Electron Scattering and CoupledChannel Calculations of $650 \mathrm{MeV}$ Proton Scattering", R.A. Mlskimen, A.M. Bemstein, G. Bernhardt, C.F. Williamson, B.A. Biown, and R. Alarcon, Phys. Rev. C 37, 1600 (1988).

4. "Magnetic Siructure of $17 \mathrm{O}$ at High Momentum", N. Kalantar-Nayestanakl, H. Baghael, W. Bertozzl, S. Dixit, J.M. Finn, C.E. Hydo-Wright, S. Kowalskl, R.W. Lourie, C.P. Sargent, P.E. Ulmer, L. Weinstein, M.V. Hynes, B.L. Berman, and J.J. Kelly, Phys. Rev. 60, 1707 (1988).

5. "Electroexcitation of the $\Delta$-Resonance in the $\left(\theta, \theta^{\prime} p\right)$ Reaction", H. Baghael. W. Bertozzi, K.I. Blomqvist, J.M. Finn, J. Flanz, C.E. Hyde-Wright, N. KalantarNayestanaki, R.W. Lourie, J. Nelson, W.W. Sapp, C.P. Sargent, P. Ulmer, L. Weinstein, B.H. Cottman, P.K. Teng, E.J. Winhold, M. Yamazaki, J.R. Calarco, F.W. Hersman, C. Perdrisat, V. Punjabl, M. Epstein, and D.J. Margaziotis, Submitted to Phys. Rev. C.

6. "K+ as a Probe of Dirac Vacuum Structure in Nuclel", N. Auerbach, G.A. Crawterd, M.B. Johnson, E. Plasetzky, and R.M. Thaler, Submitted to Phys. Rev. C. 
V. An Incomplete List of Nuclear Physics Seminars and Colloqula Given at the University of Massachusetts at Amherst from September 1, 1987 to August 31,1988

September 1: Professor Silvan S. Schweber, Brandeis University, "Quantum Electrodynamics 1927-1950: A Historical Perspective"

September 22: Dr. H. Baghaei, University of Massachusetts, "What Can Be Learned from $\left(\theta, \theta^{\prime} X\right)$ Coincidence Reactions?"

September 23: Dr. John Faulkner, Lick Observatory, University of California/Santa Cruz, "Wimps, Solar Neutrinos, and Solar Oscillations"

October 7: $\quad$ Professor Lncoln Wolfenstein, Camegie Mellon University, "Neutrino Mass, Cosmology, and the Solar Neutrino Problem"

October 8: $\quad$ Protessor Barry Holstein, University of Massachusetts, "Fundamental Symmetries in Nuclear Physics"

October 21: Professor Richard Kofler, University of Massachusetts, "Electron-Positron Annihilation at High Energies"

October 22: $\quad$ Dr. David Webb, Australlan Radiation Laboratory, "Radlation Protection Activities at the Australian Radlation Laboratory"

October 28: $\quad$ Professor David N. Schramm, University of Chicago, "Accelerator Tests of Cosmology*

November 3: $\quad$ Profesor Gerald A. Peterson, University of Massachusetts, "PEGASYS Electron Scattering Experiments at PEP"

December 1: $\quad$ Profesor Richard Kofler, University of Massachusetts, " $\theta^{+}-\theta^{-}$, TPC, and All That"

December 2: $\quad$ Professor Henry N. Wagner, Jr., Johns Hopkins Institute of Medicine, "Postron Emission Tomography"

December 3: $\quad$ Dr. R. Lourie, Massachusetts Institute of Technology, "Many-Body Effects in Coincidence Electron Scattering"

December 15: Dr. Eduardo de Rafael, Boston University and Marsellle, "Radiattve Kaon Decay and CP Violations"

December 15: Dr. Dawn Meredith, University of New Hampshire, "Chaos in a Schematic Shell Model"

January 12: $\quad$ Dr. H. Baghael, University of Massactiusetts, "A Systematlc Study of the Energy and Momentum Transfer Dependence of the ${ }^{12} \mathrm{C}\left(\theta, \theta^{\prime} p\right)$ Reaction in the Delta-Resonance Region"

February 9: $\quad$ Dr. Rlchard Hughes, Los Alamos National Laboratory, "Status of the Fitth Force"

February 11: Dr. Richard Hughes, Los Alamos National Laboratory, "Dark Matter in the Universe, In the Sun, and in the Laboratory" 
February 18: Professor Rory Miskimen, University of Massachusetts, "Pion Electroproduction at Intermediate Energies: Aro Nucleons Deformed?"

February 18: Professor Paul Boynton, University of Washington/Seattle, "Experimental Search for a Weak Intermediate-Range Force"

March 16: $\quad$ Protessor J. Dirk Walecka, CEBAF, "CEBAF: A Laboratory for Nuclear Physias"

April 7: $\quad$ Professor T. Chupp, Harvard University, "Polarized ${ }^{3} \mathrm{He}$ and Polarized Electron Scattering"

April 12: $\quad$ Professor Aron Bernstein, Massachusetts Institute of Technology, "Quasielastic Scattering in Fow Body Systems"

April 21; Professor J. Dubach, University of Massachusetts, Isospin Mixing and ParityViolating Electron Scattering or How to Determine the Electric Form Factor of the Neutron by Scattering Electrons from the Proton"

April 25: Dr. Hans de Vries, NIKHEF-K, Amsterdam, The Netherlands, "Storage Ring Plans at NIKHEF-K"

April 26: $\quad$ Dr. Richard R. Silbar, Los Alamos National Laboratory, "An interactive Interface for Beam Line Design"

April 27; $\quad$ Dr. E. Michael Rlordan, Stanford Linear Accelerator Center, "The Hunting of the Quark: A History of the MIT-SLAC Experiments"

May 4: $\quad$ Professor Normal F. Ramsey, Lyman Physics Laboratory, Harvard University, "Experiments on Time Reversal Symmetry and Partity"

May 17: Professor Jechiei Lichtenstadt, Tel Aviv University and University of Virginia,

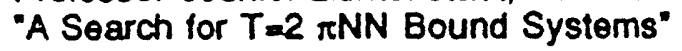

June 2:

Professor M. Stemheim, University of Massachusetts, The 3rd Conference on the intersections Between Particle and Nuclear Physics"

August 5: $\quad$ Dr. Taadaki Tamae, Tohoku University, Sendal, Japan, "Pulse Stretcher Rings arid Coincldence Experiments at Sendai"

In addition to these open formal seminars or colloquia there were weekly or biweekly meetings involving nuclear physics faculty, staff, and graduate students at which progress reports were given and current topics in nuclear physics were discussed. 


\section{Status Report on VAX Computer System}

The Digital Equipment Corporation (DEC) VAX 11/750 computing system installed in August of 1983 continues to provide essentially all of the group's computing needs. N , major failures occurred this past year; DEC maintenance covered a minor problem with the TU80 tape drive, and in-house repairs were made to the DEC LN03 laser printer following a stripped gear and a failure in the fusing unit. New hardware additions to the system, purchased with State "Educational Needs" funds, were 3 Falco 5220e video graphics terminals (which have proven to be excellent terminals) and an AST Turbolaser laser printer (shared between the group's MAC/SE and the VAX) which grovides Post Script and other options to VAX users as well as serving as a backup to the DEC LN03 laser printer which has now become an essential item for the VAX system. Two major software installations have been completed this past year. The TOPDRAWER graphics package has been implemented and is now heavily used. This package not only provides fairly sophisticated graphics capabilities, but also provides compatibility with software used at a number of other nuclear physics institutions. Secondly, the (LAMPF) $Q$ data acquisition and analysis program has been installed. This allows us to directly analyze data taken with this system at MIT-Bates and elsewhere. Finally, we have begun the process of providing network access for the VAX. This ethernet/fiber optic based network is part of a larger plan to network all departments in the Faculty of Natural Sciences and Mathematics. It will provide us with direct local access to the major computing facilities in the Engineering Computer Station and the University Computer Center and also allow us to connect to off-campus links to NSFNET and other national networks. This will eventually provide us with much improved electronic mail service, direct high-speed access to computers at the sites of our experiments, and high-speed access to national supercomputer centers. All of the necessary hardware and software has been ordered, with all costs to be paid from State sources. Installation is expected to be complete by late September. To summarize, the facilities now dedicated to the Nuclear Physics research effort include:

\section{Hardware:}


DEC VAX $11 / 750$ CPU with

$4 \mathrm{Mb}$ memory

Floating point accelerator

KU750 user-writable control store

2 RA81 $456 \mathrm{Mb}$ disk drives

1 TU80 tape drive

1 Cipher $800 / 1600$ bpi tape drive

32 asynchronous lines (2 DEC DZ11, 1 Emulex CS21)

1200 baud dial-in capability (one dedicated phone line)

link to University Computer Center

link to multi-channel analyzer

Terminals and Printers:

3 DEC VT240 graphics terminals

3 Falco 5220 e graphics terminals

2 Infoton 100 terminals

1 DEC VT101 terminal

1 TI Silent 700 series portable terminal

1 Tektronix 4006-1 graphics terminal

1 Tektronix 4010-1 graphics terminal

1 Tektronix 4013 graphics terminal

2 DEC LA120 printers

6 DEC LA36 hard-copy terminals

1 Versatec electrostatic printer/plotter

1 LVP16 color pen plotter

1 DEC LN03-plus laser printer

1 AST Turbolaser laser printer

\section{Software:}

VAX/VMS version 4.3

DOE-MACSYMA symbolic manipulation software

TEX word-processing software

TOPDRAWER graphics

$Q$ data analysis system

Comments on Computers and Computation at UMass

As was mentioned in part III.E., the retical calculations are underway in relativistic nuclear theory. Our VAX 11/750 is not powerful enough to handle that problem. Consequently supercomputer access through a DOE program has been requested for work on this problem. Also if the state budget situation permits it, the University may acquire its own supercomputer, such as a Cray. 


\section{Comments on Personnel}

During this past year there have been no changes in scientific personnel. Each of our three postdoctoral research associates (Baghaei, age 38; Rokni, age 33; Crawtord, age 29) have been with our group for over a year at the time this is being written.

Dr. Sayed Rekni has been stationed at SLAC where he has played a major role in making Monte Carlo calculations and in planning for the PEGASYS detector. He has also been involved In instrumenting the $1.6 \mathrm{GeV}$ spectrometer for the forthcoming NE-11 experiment. This experiment is scheduled to commence in earty January of 1989 and will involve the use of both the $1.6 \mathrm{GeV}$ and $8 \mathrm{GeV}$ spectrometers to simultaneously acquire data so as to separate the charge and magnetic form factors of the neutron and proton at high momentum transfer. His work has involved setting up the new ECL logic electronics, developing software for the shower counters, for the LeCroy power supply systems, for service programming, etc. He has also been involved in outlining a possible color-transparency experiment, such as $\left(\theta, \theta^{\prime} p\right)$, for PEGASYS with .

SLAC theorist Stanley Brodsky. Rokni has only retumed to the east coast to participate in the March 1988 runs on ${ }^{117} \mathrm{Sn}$ and ${ }^{41} \mathrm{Ca}$ at Bates.

Dr. Hossain Baghaei has largely been involved in software development for the deuteron threshold electrodisintegration experiment at Bates, and on the analysis of the ${ }^{117} \mathrm{Sn}$ and ${ }^{41} \mathrm{Ca}$ data acquired at Bates. He has also helped in building the new dritt chambers for use on the Bates ELSSY spectrometer. He participated in the studies at the Illinols microtron in which it was determined that the CEBAF LAS detector would have an adequate luminosity, but that some of the software used In modelling the LAS had to be improved to include large angle bremsstrahlung. He has attended CEBAF planning meetings which our faculty could not attend because of other commitments.

Dr. Gregony Crawterd, our theoretical postdoctoral research associate, has been working on the two-body relativistic problem, as well as beginning to examine relativistic effects in nuclear shell models. While Prof. Dubach is away on sabbatical leave he will be in charge of the three or four graduate students working on nuclear theory problems.

Asseciate Professor Ross S. Hicks has largely spent two summers plus his academic year on sabbatical leave at SLAC. He came back to the east coast in March to participate in the 
- $117 \mathrm{Sn}$ and ${ }^{41} \mathrm{Ca}$ runs at Bates, and he went to the Netherlands in November and in June and July to head up the ${ }^{10} \mathrm{~B}$ electron-proton coincidence experiment. While at SLAC he prepared a major portion of the Letter of Intent for the PEGASYS detector, and he he!ped organize the 1988 summer workshop related to PEGASYS. He did most of the calculations and preliminary design of the new Big Acceptance Dipole (BAD) of the PEGASYS detector, as well as outlining several experiments that may be possible using this facility. Finally he has been involved in building the new detector for the 1.6 GeV spectrometer to be used in the NE-11 experiment.

Associate Professer Jehn Dubach is taking a full-year sabbatical at CEBAF. During this period he will travel to the Soviet Union at their invitation to discuss hypernuclear physics with Soviet theorists. He will also go to Japan for thre weeks as part of the UMass-Japan collaboration mentioned in the introduction. Other faculty will go to Japan at later dates. As mentioned in the theory section, part III, he now has three graduate students working for him and Dr. Crawford. Dubach has finished several calculations during the past year, as can be seen in the list of publications, Part IV. He was in charge of the Nuclear Seminar and in charge of the VAX computer. He was vice-chairman of the 1988 Gordon Photonuclear-Reactions Conference. Assistant Protessor Rery Miskimen has built and tested new wire drift chambers for the ELSSY spectrometer at Bates during the past year. These will be used in very low counting rate experiments, the most important of which is the threshold electrodisintegration of the deuteron, for which he is spokesman. He participated in the ${ }^{117} \mathrm{Sn}$ and $41 \mathrm{Ca}$ runs at Bates, in the ${ }^{10} \mathrm{~B}\left(\theta, \theta^{\prime} \mathrm{p}\right)$ run in Amsterdam in June and July, and in CEBAF-related runs at Illinols pertaining to estimation of luminosities which should be possible with the LAS detector. Miskimen hasen at UMass for two full years now and will be subject to a thorough pre-tenure review in the fall of 1988. His tenure decision year will be the academic year 1990-1991. Other faculty supported by this grant are tenured.

Prof. Gerald Petersen participated in all runs, and oversaw the whole program.

Graduate students in nuctear experiment. who worked in our group this past summer are Marc Frodyma, who is located at SLAC, Alina Cichockl, Peter Morgan, and Li-Juan Wol. Of these students, only Frodyma has passed the qualifying examinations. It is hoped that he can finish his thesis work in 1989. The other students have not taken their qualifying exams yet. it is likely that 
some may not pass their exams, or that they will continue in nuclear physics. At this point our group lacks experienced and dedicated graduate students in experimental nuclear physics. Of the four theoretical nuclear physics students (Christepher Arabadlis, Joe Gallant, Geoffrey Feldman, and Paul Nakroshis), the first three have passed their qualifying exams. Only one student, Arabadjis, is being supported by the DOE grant. This group of theory students is the first to be directed by Professor Dubach. From his arrival at UMass in September of 1980 through May of 1987, Dubach had no graduate students under his direction. Now he has several students, whereas the situation with regard to the experimental students is somewhat shaky. 

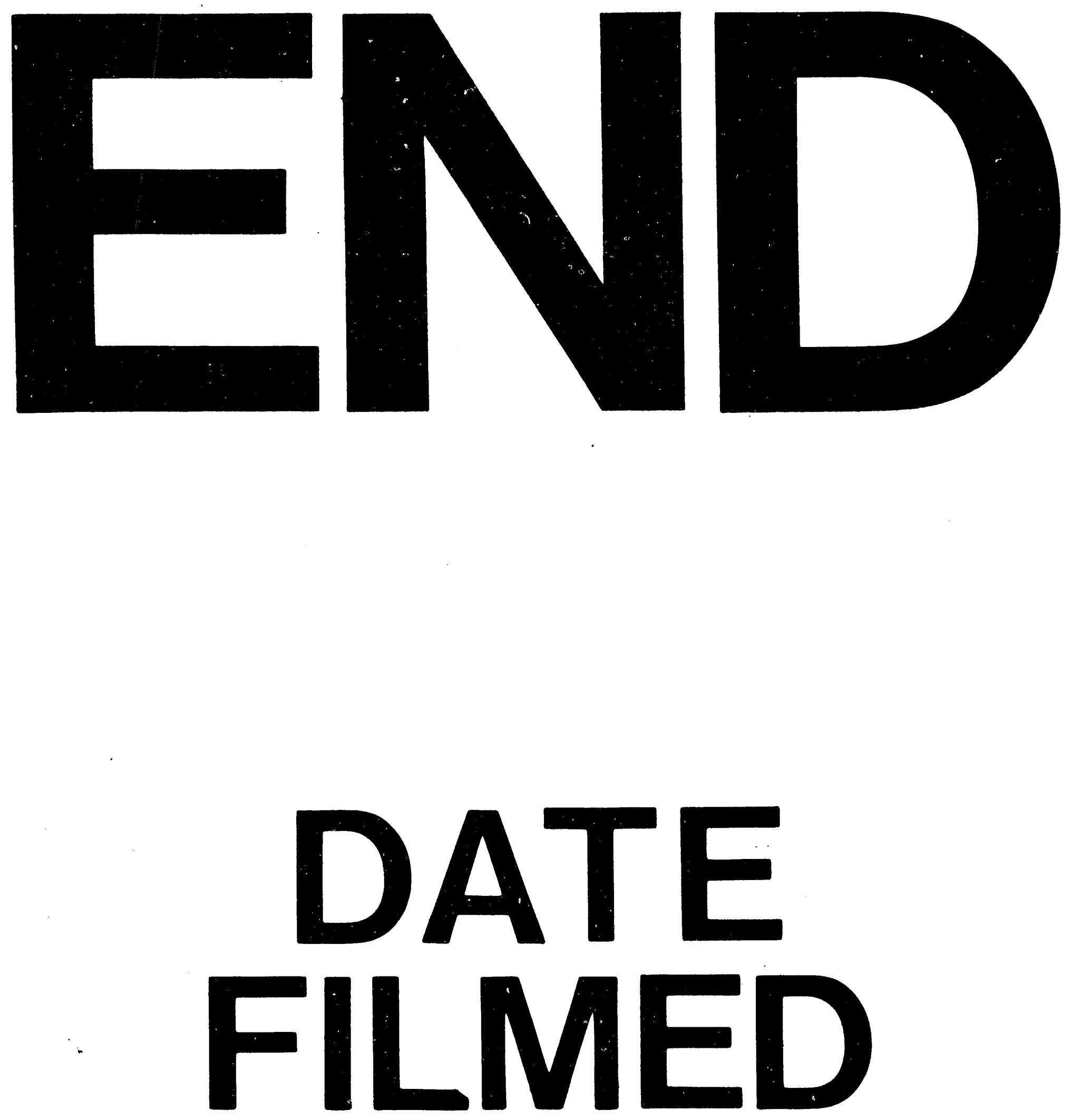

1

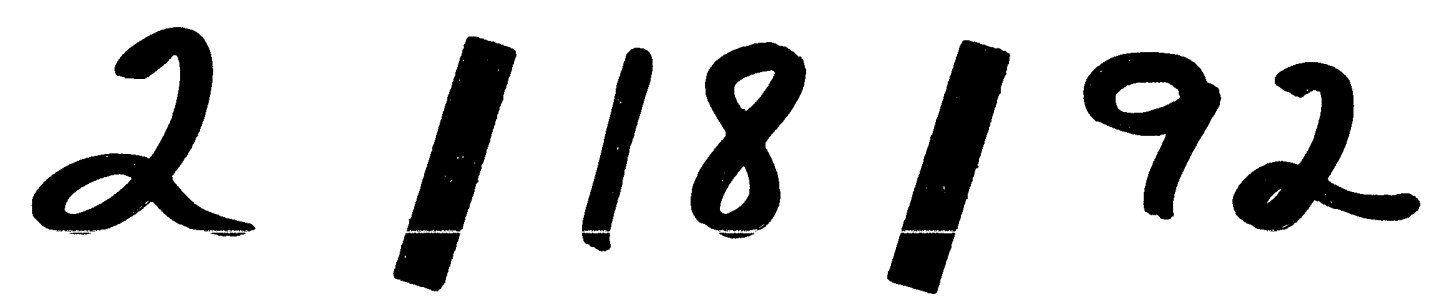


